\title{
A Sector-Based Approach To Hybrid TDMA And NOMA With Digital Beamforming For Fairness Improvement In Wireless Powered Communication Networks
}

\section{Deukong Yoon}

Hanyang University - Seoul Campus: Hanyang University

\section{Mwamba Kasongo Dahouda}

Hanyang University - Seoul Campus: Hanyang University https://orcid.org/0000-0003-0376-683X Juhyun Maeng

Hanyang University - Seoul Campus: Hanyang University Inwhee Joe ( $D$ iwjoe@hanyang.ac.kr)

Hanyang University - Seoul Campus: Hanyang University https://orcid.org/0000-0002-8435-0395

\section{Research}

Keywords: Time-Division Multiple Access, Non-Orthogonal Multiple Access, fairness, sum-throughput

Posted Date: July 1st, 2021

DOl: https://doi.org/10.21203/rs.3.rs-568919/v1

License: (c) (1) This work is licensed under a Creative Commons Attribution 4.0 International License. Read Full License 


\title{
A Sector-based Approach to Hybrid TDMA and NOMA with Digital Beamforming for Fairness Improvement in Wireless Powered Communication Networks
}

\author{
Deukgong Yoon, Mwamba Kasongo Dahouda, Juhyun Maeng and Inwhee Joe*
}

\footnotetext{
${ }^{*}$ Correspondence:

iwjoe@hanyang.ac.kr

Department of Computer Science,

Hanynag University, Seoul 04763,

South Korea

Full list of author information is

available at the end of the article
}

\begin{abstract}
In recent years, Wireless Powered Communication Network (WPCN) has been a promising technology that can be applied to existing low-power sensor networks and the Internet of Things (IoT). Sensor nodes or loT devices are usually battery-powered. It is possible to use naturally collectable energy such as solar and wind without using a battery, but this is not a stable supply of energy. Therefore, the idea of operating a sensor network by separately setting a base station that continuously supplies power with radio waves has been presented. ThMris paper proposes an approach for how to combine Non-Orthogonal Multiple Access (NOMA) and Time-Division Multiple Access (TDMA) among various multiple access protocols applicable to wireless powered communication networks. There are some problems using TDMA alone. If a time slot is allocated so that the sum-throughput is maximized, the fairness of nodes is not guaranteed. To cope with these shortcomings, NOMA, which is known as a method to improve fairness, is mixed. Our approach is that cells are divided into sectors so that TDMA is used among sectors while NOMA is used within sectors. In addition, optimization of the sector by sector time allocation for maximum sum-throughput can lead to residual energy in certain sectors. Therefore, a directional digital beamforming adapted to the transmission for each sector is used for efficient energy transmission. Unlike the previous user clustering, we attempt to generalize the number of nodes for NOMA from the fixed two nodes to any nodes by introducing the sector-based system model. The simulation results show that there is a trade-off between the sum-throughput and fairness because the sum-throughput increases but the fairness decreases as the number of sectors increases. As a result, we can suggest that a balanced range lies in between three and six sectors to satisfy both the sum-throughput and fairness at the same time. Finally, it is proven that our hybrid approach improves fairness significantly with the increasing number of nodes, as compared to the original TDMA only.
\end{abstract}

Keywords: Time-Division Multiple Access; Non-Orthogonal Multiple Access; fairness; sum-throughput

\section{Introduction}

\subsection{Research Background}

The wireless powered communication networks (WPCN) basically proposes a new paradigm based on sensor networks. Therefore, understanding the sensor network makes WPCN more realistic [1]. Sensors are distributed over a large area to mon- 
itor the area. Information to be monitored can be temperature, humidity, sound pressure, light, and image information. The information collected from various regions is collected and processed to extract meaningful information. You can also track moving objects near the sensor, detect forest fires, and use a magnetic sensor to check for the presence of a nearby vehicle. There are also sensors that are smaller in size than the coins from the size of the shoe box. It is possible to have each sensor communicate with each other, which in this case resembles an ad-hoc network. The zigbee protocol enables routing between nodes. If it is not an ad-hoc network, one sink node collects data, and if the sink node is connected to the Internet, you can easily view the information that the sensor network is monitoring over the Internet. Since most sensor networks use the IEEE 802.15.4 protocol, refer to the relevant specifications for detailed PHY, MAC, and frames. The sensors can be equipped with a small and lightweight operating system or can operate with only the firmware without the operating system. The information monitored by the sensor can be sent periodically, or it can be sent only when a certain event occurs. Because of this, theoretically, when measuring performance by modeling a sensor network, the analysis contents are determined depending on whether to target the node that transmits information periodically or always or only when a specific event occurs. It may vary. Since multiple nodes need to use the channel together, multiple access is essential. In this case, if the information periodically occurs, multiple access based on a non-competitive reservation based on TDMA is appropriate, and if it is transmitted whenever a specific event occurs, it is not reserved. Instead, multiple access based on the competition using the channel is appropriate. The sensors covered in this paper always deal with sensors that have information to send. It is assumed that every node always carries information. In WPCN, depending on whether the capacity of the battery is large or small, the amount of energy harvested is large and small, and unlike existing networks, there may be insufficient data to send, but energy may be sufficient to have no data to send. Therefore, appropriate protocol should be used depending on where to use it.

WPCN eliminates the need for frequent manual battery replacement, and thus significantly improves the performance over conventional battery-powered communication networks in many aspects, such as higher throughput, longer device lifetime, and lower network operating cost [2]. There are three types of wireless charging methods as shown in Fig. 1; the method of charging by contacting within five centimeters $(5 \mathrm{~cm})$ is called inductive coupling. And the method to wirelessly charge to a medium distance from 10 to $50 \mathrm{~cm}$ is called magnetic resonant coupling. Finally, the method that can be charged to the farthest distance, more than one meter, is electromagnetic radiation, and WPCN uses this far-discharge method. It is difficult to generalize rather than use all the products on the market, but powercast's products used in the lab showed almost no performance when it exceeded two meters $(2 \mathrm{~m})$. If the antenna is used as a dipole antenna rather than a directional antenna, the chargeable distance is further reduced. At this time, the power of the wireless charger is limited to three watt $(3 \mathrm{~W})$, because it is dangerous to the human body. If the charger can be powered up, the charging distance will be longer. 


\subsection{Research Needs}

Battery-powered low-power wireless communication devices (sensors) must be periodically charged or replaced. Therefore, if you set aside a power station that charges power with radio waves and add a rectifier to collect wireless power to the wireless communication device, the wireless communication device can collect energy. In [3], WPCN looks very useful and promising, but there is one problem that is not easy to solve; because radio waves exponentially decrease in distance when propagating in space, the distance that the energy can be collected from a distant communication device decreases compared to a nearby communication device. Moreover, with the collected energy, the information must be transmitted to the access point (AP), the propagation property that decreases in distance is adversely affected; this problem is called the Doubly-Near-Far problem. To maximize the performance of WPCN, simply mathematically if resources are allocated to maximize sum-throughput, resources are allocated very unfairly due to the DoublyNear-Far problem. As a method to alleviate this problem, a method of mixing with NOMA is proposed. In the next section, we explain why NOMA mixed can alleviate the inequality problem by explaining the nature of NOMA. Furthermore, digital beamforming is introduced to prevent unnecessary power transmission in accordance with optimal resource allocation for maximizing sum-throughput. Since digital beamforming enables power transmission in multiple directions at the same time, efficient power transmission is possible to all nodes in the WET.

The paper is organized as follows: section 2 presents related work, and section 3 gives an overview of the system model; section 4 presents the simulation results. In section 5 , we give our conclusion and future work.

\section{Related Work}

\subsection{Harvest-Then-Transmit}

The nodes in a WPCN can be equipped with hardware capable of harvesting energy from wireless signals, that is, their battery can be ubiquitously recharged without physical connections [4]. In [5], Rui Zhang's paper is one of the successful paper on maximizing the throughput of WPCN, it analyzes the performance of hybrid access point (H-AP) and multiple nodes that can perform energy charging and information collection. The basic network of WPCN is shwon in Fig. 2.

In [6], energy beamforming was optimally designed for a WPCN in which multiple antennas were equipped at the Energy Transfer and user cooperation between two user nodes. In [7], a "harvest-then-cooperate" protocol was proposed in which the user nodes and their dedicated relay nodes harvest energy and then cooperatively work to transmit the information of the user node to improve the fairness was considered. The "relay" user node placement and the user node's communication mode selection problems were also discussed. In [8], the paper considered two communication groups that cooperated via the wireless power transfer (WPT) and time sharing to fulfill their expected information delivery and achieve a "win-win" collaboration. Communication and energy scheduling can also be performed in the spatial domain when energy node and access point are equipped with multiple antennas [9]. Based on the Fig. 2, when the H-AP operates in a half-duplex that cannot simultaneously charge and collect energy, the given frame should be divided into a wireless energy 
transfer (WET) and a wireless information transmission (WIT). The length of one frame is called $\mathrm{T}$ and the frame is repeated. This is illustrated in the Fig. 3 below.

The WET and WIT of time was allocating in [3], it was named harvest-thentransmit (HTT) protocol. It was intended to show in [3] that each node has a time slot to maximize throughput after initially transmitting energy to the node through WET. Through this scheduling, the throughput is maximized, but time slots are allocated very unfairly. In [3], an additional perfectly fair resource allocation is also provided, but this reduces a large amount of throughput. In WPCN, the maximum throughput is theoretically the same when maximizing throughput regardless of the multiple access (MA). In the author's existing research [5] NOMA and TDMA showed the same throughput. However, the throughput region may have a wider range of specific multiple access (MA) techniques than other techniques. In this case, there is a difference in fairness.

\subsection{Non-Orthogonal Multiple Access}

There are several types of NOMA, but the most frequently used is the power domain NOMA. Unless otherwise specified, the NOMA in this paper refers to the power domain NOMA.

\section{1) Decoding conditions}

In the case of NOMA, the signal is decoded using the difference in power. For example, when the quadrature amplitude modulation (QAM) is used, if s1, s2, and s3 signals are received at the same time, they are expressed on the signal constellation as shown in the Fig. 4.

The method of decoding the superimposed signal is as follows: First, the entire signal is decoded. Since the whole signal is in the first quadrant, for example QAM. It decodes into the symbol of the first quadrant. In addition, s1 is estimated as a channel gain value estimated through channel estimation. After subtracting the estimated $\mathrm{s} 1$ from the total signal, the $\mathrm{s} 2+\mathrm{s} 3$ signal remains. The signal is decoded again. Since it is in the first quadrant once again, it is decoded into the symbol of the first quadrant. Then, s2 is again estimated and subtracted from the remaining signal. Then, the remaining s3 signal remains and this signal corresponds to the quadrant; therefore, decoding with this symbol completes the self-interference cancellation (SIC) process as described in Fig. 5. At this time, there is a condition that decoding can be sufficiently performed only when the strength of signals (SNR, strictly speaking) differs exponentially [10].

For example, if the signal of s3 has an SNR of a, s2 should have the strength of $\mathrm{ab}$ and $\mathrm{s} 1 \mathrm{of} a b^{2}$. It is explained in the formula as follows :

$$
\begin{aligned}
& \frac{P_{1} h_{1}}{P_{2} h_{2}+P_{3} h_{3}+N}>S I N R_{t h} \\
& \frac{P_{2} h_{2}}{P_{3} h_{3}+N}>S I N R_{t h}
\end{aligned}
$$




$$
\frac{P_{3} h_{3}}{N}>S I N R_{t h}
$$

As it will be described later, simply determining this decoding success condition as a difference of SNR has no problem when the absolute value of the signal strength is small, but when the absolute value of the signal strength is large, it is difficult to understand intuitively. For example, if the difference in SNR should be 10 or more, it is expressed as follows.

$$
\frac{P_{1} h_{1}}{N}-\frac{P_{2} h_{2}}{N}>10
$$

When the absolute value is small, decoding can be performed when the value of $S N R_{1}=\frac{P_{1} h_{1}}{N}>12$ and the value of $S N R_{2}$ is 1 . It can be decoded even when checked on the signal space. However, as an extreme example, if the value of $S N R_{1}$ is 1000 and the value of $S N R_{2}$ is 980 , considering from the signal space, if these signals point in different directions, there is a high probability that the sum of the signals will go to different quadrants. Still, decoding is possible according to the conditions. Therefore, the decoding condition must be set as a ratio.

\section{2) Fairness performance}

Earlier, it was said that the power intensity must be sufficiently different in order to succeed in decoding. User cooperation is another method to improve fairness performance. User cooperation helps the user node that harvested more energy to relay the information of the user nodes that have less energy or weak transmission channel states [11]. In WPCN, the power intensity is significantly different because the intensity of the radio waves (both energy transfer and information transfer) decreases exponentially over distance. Using this point, NOMA can be successfully applied. TDMA from the author's previous study [9], and NOMA fainress were analyzed. The power intensity is affected and changed only by the distance, not the orientation, the nodes distributed in a circular shape can be arranged on a straight line. In the Fig. 6 below; it is understood that the nodes are distributed close to each other and, if expressed quantitatively, the standard deviation of the distance is small.

In this situation, the fairness of TDMA is high. On the other hand the fairness of NOMA has a small value. When the standard deviation has a large value due to different circumstances as described in Fig. 7; the fairness of the NOMA is high and the TDMA has a low value.

In [9], the fairness change of TDMA and NOMA was examined while linearly increasing the value of the standard deviation, and it was confirmed that NOMA increases and TDMA decreases gradually. In reality, it is reasonable to assume that nodes are uniformly distributed, so the fairness of NOMA in WPCN can be seen as good compared to other multiple accesses. This is because the radio waves attenuate with distance and can use this difference to decode the signal. Another way to interpret this phenomenon is to think of it as a capacity region. 
In the case of Fig. 8, the values of $R_{1}$ and $R_{2}$ are determined at the intersection between the thick and thin lines. It can be said to be the focus of CD. In this case, the values of $R_{1}$ and $R_{2}$ are not just. On the other hand, if NOMA is used successfully, there is a possibility of theoretically having throughput somewhere between $\mathrm{C}$ and $\mathrm{B}$, which improves fairness.

\subsection{Clustering with NOMA}

Clustering of nodes plays an important role in conserving energy of wireless sensors networks (WSN) [15], and clustering algorithms have been widely used to reduce energy consumption. In [12], it mentioned that NOMA can be applied to WPCN to improve fairness, for the first time, a method of grouping two nodes into one cluster and using NOMA in a cluster and TDMA between clusters has been proposed. As the number of nodes increases, the number of cases in which two nodes can be paired increases and it is optimal. A lot of computation is required to obtain a clustering combination that can achieve throughput. Therefore, a sub-optimal method of sorting nodes by distance, and then matching the closest node to the middle node, then the closest node to the next middle node has been studied. However, in [13], the number of nodes applying NOMA is limited to two. That is, only two nodes use resources in a non-orthogonal manner and calculate the number of cases in which node belongs to which clustering to achieve optimal performance. On the other hand, this study generalizes the number of nodes belonging to a cluster to two or more, calculates only the sectors, and analyzes them.

\section{System Model}

We present in Fig. 2 some basic building blocks of WPCN. In a WPCN, energy nodes transmit wireless energy to wireless devices in the downlink, and the wireless devices use the harvested energy to transmit their own information to the access point (AP) in the uplink. A point to notice is that a wireless node can in fact operate in an information/energy full-duplex manner, which is able to transmit information and harvested energy to/from the AP/energy node (H-AP) in the same band [14]. The environment of nodes and H-AP configurations assumed in this paper are shown in Fig. 9. The nodes are randomly distributed in a circular shape around the H-AP.

The positions of the nodes are expressed on the x,y plane, $x=r \cos (\theta)$, $y=r \sin (\theta)$, where $r \sim U(0.5,9.5), \theta \sim U(0,2 \pi)$. The reason that the position is distributed from $0.5 \mathrm{~m}$ is that if the distance is too close to 0 , the throughput is theoretically too high. The user is represented by $U_{i}, i=1, \ldots, K$. In our system model, we consider a digital beamforming with multiple-input and single-output (MISO) where the H-AP is equipped with multiple antennas, and each node is equipped with a single antenna.

\section{Proposed Method}

Our method divides cells into sectors, with TDMA used within sectors and NOMA used inside sectors. The H-AP and all nodes use a directional antenna. H-AP simultaneously transmits power for each sector through digital beamforming. Nodes transmit information to the $\mathrm{H}-\mathrm{AP}$ according to the amount of energy received. The channel gain between $\mathrm{H}-\mathrm{AP}$ and user $U_{i}$ is represented by $h_{i}$, and it is assumed that 
the channel gain when sending information to $\mathrm{H}-\mathrm{AP}$ and receiving energy from $\mathrm{H}-$ AP is the same assuming reciprocity. It is assumed that the channel gain is constant without changing the channel gain for each frame using the quasi-static flat-fading model. In addition, it is assumed that H-AP can fully understand channel state information (CSI). The circular shape is divided into sectors. TDMA is applied between sectors. The allocation of WET and WIT in the TDMA time slot is basically the same as in Fig. 2. However, here, one node does the WIT in one slot, and then an other node in an other sector does the WIT as well. When doing WIT, all nodes in the sector simultaneously transmit information using NOMA. The bolded part in Fig. 8 shows the sector doing WIT. As shown in Fig. 2, the time to perform WET is $\tau_{0}$. And the remaining time for WIT intervals are denoted by $\tau_{i}$ at this time. The energy collected by the H-AP can be expressed by equation (5).

$$
\begin{aligned}
& \sum_{i=0}^{n} \tau_{i}=T=1 \\
& E_{i}=P_{o} h_{i} \tau_{0}, i=1, \ldots, K
\end{aligned}
$$

At this time, $P_{0}$ represents the WET power of H-AP. The energy collection efficiency is assumed to be 1 for simplicity. The power transmitted by node to H-AP using the collected energy is :

$$
P_{i}=\frac{E_{i}}{\tau_{j}}=\frac{P_{0} h_{i} \tau_{0}}{\tau_{j}}
$$

Where, $\mathrm{j}$ represents the index of the sector. As discussed earlier, the decoding conditions in NOMA is that multiple nodes within a sector must be decoded using SIC. At this time, you should not simply use the difference in signal-to-noise ratio (SNR), but use the ratio of SNR and, the expression is as follows :

$$
\frac{S N R_{i}}{\sum_{k=i+1}^{n_{i}} S N R_{k}+1}>S I N R_{t h}
$$

Where, i denotes the index of the node in the $j^{t h}$ sector, and $n_{i}$ is in the $j^{\text {th }}$ sector. The relation $S N R_{i}=\frac{P_{i} h_{i}}{N}$ indicates the signal to noise ratio. We assume that $\mathrm{N}$ is sorted in big order. $\mathrm{N}$ is the noise power. This is expressed by generalizing equation (1) of the decoding conditions. The throughput $R_{i}$ that can be generated in the $j^{\text {th }}$ sector is calculated only up to the node that satisfies the decoding condition, and the following equations are calculated when decoding is successful. If decoding fails from the first strongest signal, $R_{i}$ has a value of 0 .

$$
S N R_{j}=\sum_{i=1}^{n_{j}}\left(\tau_{j} \log \left(1+\frac{S N R_{i}}{\sum_{k=i+1}^{n_{j}} S N R_{k}+1}\right)\right)
$$




$$
\begin{aligned}
& S N R_{j}=\tau_{j} \log \left(1+\sum_{i=1}^{n_{j}} S N R_{i}\right) \\
& S N R_{j}=\tau_{j} \log \left(1+\sum_{i=1}^{n_{j}} \gamma_{i} \frac{\tau_{0}}{\tau_{j}}\right) \\
& S N R_{j}=\tau_{j} \log \left(1+\Gamma \frac{\tau_{0}}{\tau_{j}}\right)
\end{aligned}
$$

$\gamma_{i}=\frac{P_{0} h^{2} i}{N}$ is a constant value, it can be simplified like the last expression, which makes it easy to check that it is a convex function. Therefore, the sum-throughput of the network can be expressed as follows :

$$
\begin{aligned}
& R_{\text {sum }}=\sum_{j=1}^{n} R_{j} \\
& R_{\text {sum }}=\sum_{j=1}^{n} \tau_{j} \log \left(1+\Gamma \frac{\tau_{0}}{\tau_{j}}\right)
\end{aligned}
$$

After optimizing the transmission time for each sector for maximizing the sumthroughput, a small amount of time is allocated to the sector with a low transmission rate, so the energy transmitted by the H-AP during WET to the nodes in the sector may be wasted. Therefore, the H-AP periodically varies the energy allocation for each sector with variable digital beamforming adapted to the amount of information received for each sector. As a result, the optimization results in, less power is transmitted in a sector allocated with a small transmission time, and a large amount of energy is transmitted in a sector allocated with a large amount of time, thereby minimizing unnecessary energy transmission. H-AP operates as a half-duplex that cannot simultaneously transmit energy and collect information, and frames are divided into wireless energy transfer (WET) and wireless information transmission (WIT). The lengths of WET and WIT in one frame are $T_{1}$ and $T_{2}$ respectively, and each $\tau_{k}$ is the DL and UL time per sector. H-AP determines the length of the WET according to the time allocated to the sector with the highest transmission rate as it is shown in Fig. 10.

\subsection{Throughput Maximization}

The indicators to be compared in this paper are maximum sum-throughput and fairness at a given time. The variable that is adjusted to obtain the maximum throughput is the length of the time slot $\tau_{i}, j=1, \ldots, n$. This can be specified as the following optimization problem : $\max \tau R_{\text {sum }}$

$$
\text { s.t. } 0<\tau_{j}<1, \text { for } j=1, \ldots, n
$$

$$
\sum_{j=1}^{n} \tau_{j}=1
$$


When solving the optimization problem, defining which class belongs to make it easy to predict the results and to verify the results when numerically calculated. In the case of linear or convex optimization problems, it is known that an optimal value can be effectively and quickly found by applying well-known algorithms (for example, simplex or interior-point). In particular, in the case of convex optimization, since the local minimum is the global minimum, it is good to check whether the optimization problem belongs to convex or not. Looking at the above problem, if all the constraints are linear, proving that only $R_{\text {sum }}$ is a convex function becomes a convex optimization problem. The easiest way to prove that it is a convex function for a single variable function is to find the second derivative and have a value greater than zero. However, in the case of a multivariate function, the value of $\nabla^{2} f(x)$ called Hessian matrix is computed and the random vector $v$ is the product to check whether the value of $v^{T} \cdot \nabla^{2} f(x) \cdot v$ is greater than or equal to 0 (or less). In fact, it is note worthy that the method of mixing TDMA and NOMA coincidentally has the same function as the HTT method calculated in [2]. So the proof process is the same. Here, we will develop the formula in more visual detail while maintaining the matrix form. If we first obtain the Hessian matrix from $R_{j}(\tau)$.

$$
\begin{aligned}
\nabla^{2} R_{j}(\tau)=\left[\begin{array}{ccccc}
\frac{\partial^{2} R_{j}}{\partial \tau_{0}^{2}} & \cdots & \frac{\partial^{2} R_{j}}{\partial \tau_{0} \partial \tau_{j}} & \cdots & \frac{\partial^{2} R_{j}}{\partial \tau_{0} \partial \tau_{n}} \\
\vdots & \frac{\partial^{2} R_{j}}{\partial \tau_{1} \partial \tau_{1}} & \cdots & \cdots & \vdots \\
\frac{\partial^{2} R_{j}}{\partial \tau_{j} \partial \tau_{0}} & \vdots & \ddots & \ldots & \vdots \\
\vdots & \vdots & \vdots & \ddots & \vdots \\
\frac{\partial^{2} R_{j}}{\partial \tau_{n} \partial \tau_{0}} & \cdots & \ldots & \cdots & \frac{\partial^{2} R_{j}}{\partial \tau_{n} \partial \tau_{n}}
\end{array}\right] \\
\nabla^{2} R_{j}(\tau)=\left[\begin{array}{ccccc}
-\frac{1}{\ln 2} \frac{\Gamma_{j}^{2} \tau_{j}}{\left(\tau_{j}+\Gamma_{j} \tau_{0}\right)^{2}} & \cdots & \frac{1}{\ln 2} \frac{\Gamma_{j}^{2} \tau_{0}}{\left(\tau_{j}+\Gamma_{j} \tau_{0}\right)^{2}} & \ldots \\
\vdots & \ddots & \ldots & \vdots \\
\frac{1}{\ln 2} \frac{\Gamma_{j}^{2} \tau_{0}}{\left(\tau_{j}+\Gamma_{j} \tau_{0}\right)^{2}} & \cdots & -\frac{1}{\ln 2} \frac{\Gamma_{j}^{2} \tau_{0}^{2}}{\tau_{j}\left(\tau_{j}+\Gamma_{j} \tau_{0}\right)^{2}} & \vdots \\
\vdots & \ldots & \ldots & \cdots
\end{array}\right]
\end{aligned}
$$

The size of the matrix is $\mathrm{n} \mathrm{x}$, and wherever a value is not specified, it has a value of 0 . Only 4 places in the matrix have non-zero values because of this product $v^{T} \cdot \nabla^{2} R_{j}(\tau) \cdot v$

$$
\begin{aligned}
& v^{T} \cdot \nabla^{2} R_{j}(\tau) \cdot v=\frac{1}{\ln 2} \frac{\Gamma_{j}^{2}}{\left(\tau_{j}+\Gamma_{j} \tau_{0}\right)^{2}}\left(-v_{0}^{2} \tau_{j}+2 v_{0} v_{j} \tau_{0}-v_{j}^{2} \frac{\tau_{0}^{2}}{\tau_{j}}\right) \\
& v^{T} \cdot \nabla^{2} R_{j}(\tau) \cdot v=-\frac{1}{\ln 2} \frac{\Gamma_{j}^{2}}{\tau_{j}\left(\tau_{j}+\Gamma_{j} \tau_{0}\right)^{2}}\left(v_{j} \tau_{0}-v_{0} \tau_{j}\right)^{2} \\
& v^{T} \cdot \nabla^{2} R_{j}(\tau) \cdot v=\leq 0
\end{aligned}
$$


Since the constant part is always greater than 0 and the squared part is also equal to or greater than 0 , all this is a convex function because the whole is less than or equal to 0 . Because the addition of the convex function is again a convex function, $R_{\text {sum }}$, which is the sum of con, is a convex function; therefore, the optimization problem in equation (8) is a convex optimization problem and the optimal value can be efficiently obtained numerically.

\subsection{Jain's Fairness}

Jain's fairness index is often used as an indicator of fairness. The method of calculating fairness using throughput $R_{j}$ of each node is as follows:

$$
J_{\text {ain's }}^{\prime} \text { Fairness }=\frac{\left(\sum_{i=1}^{K} R_{i}\right)^{2}}{K \sum_{i=1}^{K} R_{i}^{2}}
$$

Using this index, the more different values are, the closer to the $1 / \mathrm{K}$ value is, and if they have completely the same value, a value of 1 can be obtained. This index cannot compare fairness with absolute values when the number of nodes is different. When the number of nodes is 2 , even if it is not correct, it has a value of 0.5 , which cannot be directly compared to the value of 0.5 when the number of nodes is 10 . Therefore, it is possible to obtain a meaningful result only by making a relative comparison under the same conditions.

\section{Results and discussion}

\subsection{Experimental Environment}

Experimental research contains dependent and independent variables. The dependent variables are the variables being manipulated, and the moment model was solved in Matlab environment. The experiment was carried out in order to determine Sum-Throughput and Fairness; many factors affect the simulation environment, including the number of sectors, the number of nodes, and the distance between nodes and hybrid access point, as well as whether or not thresholds are used. Throughout all of the simulations, the noise power value was kept steady.

\subsection{Experimental Results and discussion}

The moment model was solved in Matlab environment. In order to achieve reliable investigation by simulation, we set many parameters and we did twice the simulation; with a threshold and without a threshold. Thresholds allow us to alert analysts to data that is likely to be relevant to their decision-making. Thresholds enable us to attract rapid attention to data depending on expression evaluation results. We used several values of Threshold to assess the Sum-throughput according to Wireless Energy Transfer. As a consequence, in order to better interpret our system model described in section 3, Fig. 9; we first experimented without applying the threshold, and then we applied the threshold and conducted different evaluations.

\subsubsection{SINR Threshold Not Applied}

The SINR threshold is not applied first, the model was compared with the theoretical value to verify the model without applying the SINR threshold. When the 
number of nodes is 100 and the number of sectors is changed from 1 to 15 , the performance of Hybrid TDMA and NOMA proposed in this paper was measured.

The nodes are randomly distributed as shown in Fig. 11. The middle triangle points to the H-AP and one circle points to one node. It was drawn using MATLAB scatter function. Each time, the maximum sum-throughput is measured, the arrangement of nodes is randomly redistributed and the process is repeated 500 times to average.

Looking at the maximum sum-throughput first, it can be seen from Fig. 12 that this value does not change even if the number of sectors changes. This value is consistent with the value obtained by HTT, which confirms that the maximum sum-throughput is theoretically the same. The SINR threshold is not applied in this simulation. When a threshold is applied, a node that fails to decode occurs, and the throughput is lower than the theoretical value. In the case of fairness, there are three fairnesses. As each fairness is closer to 1 , the time value allocated between the sectors is similar, and thus the transmission rate per sector becomes similar. On the contrary, as the distance from 1 increases, the more time values are allocated to a specific sector, resulting in a difference in the transmission rate for each sector. This is because time allocation for each sector was optimized to maximize the sum-throughput of the sum of the transmission rates for each sector. In Fig. 13, the between-sector measured the fairness between sectors by obtaining the throughput of one sector. The in-sector is a measure of the fairness of the throughput of nodes in one single sector. Finally, all-node is a measure of fairness considering the throughput of all nodes.

As the number of sectors increases, the fairness between sectors and the fairness of all nodes decreases, and the fairness within a sector increases little by little. This is due to the nature of optimization that tries to allocate more time resources to high-throughput sectors. If the sector is divided by the same number as the number of nodes and one node is included in one sector, this becomes the same as multiple access using only TDMA. Therefore, as the number of sectors increases, the properties of NOMA become weaker and the properties of TDMA become stronger, thereby reducing the fairness of all-nodes. When the number of sectors is 1 , it is the same as using only NOMA than fairness comes out the highest. However, this means that 100 nodes are trying to transmit information at the same time, and if the threshold is applied, throughput is rarely displayed. When considering the throughput of nodes in a sector, the tendency for fairness to increase is that as the number of sectors increases, the number of nodes in a sector decreases, which seems to be because the minimum value of jain's fairness has $1 / \mathrm{K}$. As the number of nodes in a sector decreases, the minimum value also increases, and thus it appears that the fairness increases.

\subsubsection{SINR Threshold Applied}

The simulation results are different when applying the threshold, the number of nodes was limited to 30 , if the number of nodes is too large, the probability of decoding is increased. First, this time, the number of sectors was fixed and the number of nodes per sector was the same. Two cases were plotted. Sum-throughput of 5 and 15 sectors are shown in Fig. 14, and in Fig. 15, the sum-throughput are 
shown according to the change in WET power. After several simulations, statistics were processed. If we have 30 nodes and divide the sector into 5 , there will be 6 nodes in one sector. When a sector is divided into 15, two nodes are included in one sector. As it can be easily predicted, since NOMA is used in one sector, the more nodes are included, the more difficult decoding is expected and throughput is reduced. Simulation results confirm this. When the number of sectors is 5, even a SINR threshold of about $3 \mathrm{~dB}$ showed a significant reduction in throughput compared to when there is no threshold; larger the threshold value is, big is the reduction.

On the other hand, if you look at the result in Fig. 14, which includes two nodes in one sector.

Decoding was performed without difficulty, so the reduction in throughput was small. For comparison, the y-axis scale was the same in both cases as described in Fig. 15.

Fig. 15 shows the reason why the difference of throughput according to the size of the SINR threshold is that the amount of information that can be received by the $\mathrm{H}-\mathrm{AP}$ varies depending on the threshold. That is why, if the threshold decreases, the amount of information received by the H-AP increases as the number of decoding increases. Conversely, as the threshold increases, the amount of information received by the H-AP decreases as the number of decoding decreases. Fig. 16 shows a graph of changes in sum-throughput and fairness when the number of sectors is changed. The environment is the same as in Fig. 12.

One thing to mention is that you cannot choose the number of sectors with optimal performance. When the sum-throughput is high, the fairness decreases, and when the sum-throughput is low, the fairness increases. When the number of sectors is 1 , the sum-throughput is too low, and when the number of sectors is high, the fairness is too low. As described above, if the number of sectors is small, the number of sectors becomes NOMA, and TDMA is characterized by a larger number of sectors, this time the threshold is applied. So you need to find the right compromise. In mobile communication, three sectors are usually used. In Fig. 16 above, when the number of sectors is 3 , sum-throughput and fairness intersect, so selecting the number of sectors near this part can guarantee both performances to some extent. It can be selected according to the needs of the application. Another thing to mention is that the absolute value of fairness seems low because jain's fairness index has a minimum value of $1 / \mathrm{K}$ depending on the number of nodes. Conversely, if the number of nodes being tested is small, the fairness value may appear high. Here, you only need to make a relative comparison according to the number of sectors.

Lastly, in order to verify the model once again, we compared the fairness result obtained when using a model that proposed the fairness result from applying the sub-optimal scheme in [5]. As shown in Fig. 16, there is a trade-off between the sum-throughput and fairness, because the sum-throughput increases but the fairness decreases as the number of sectors increases. The sum-throughput and fairness cross over at three sectors, and after six sectors, the sum-throughput is not changed much but the fairness degrades substantially with the increasing number of sectors. As a result, we can suggest that a balanced range lies in between three and six sectors to satisfy both the sum-throughput and fairness at the same time.

We have to make sure to include 2 in the sector and linear distance $\left(d_{i}=i_{m}\right)$ by arranging and comparing the nodes, we can verify the model in that the two 
models should in fact have the same results. However, in the threshold condition [5], there is a limitation in that numerical values cannot be exactly matched, even if the parameters are set equally, because the difference in SNR is used. In Fig. 17, the fairness was calculated by the method proposed in the paper, and the value was slightly smaller. The overall trend is similar. Therefore, it can be seen that the simulation was relatively good. In our system model, the nodes are randomly distributed in a circular shape. The circular shape is divided into sectors and then the TDMA is applied between sectors. In TDMA each node is allocated a time slot within time frame.

In Fig. 18, we have calculated and compare the fairness between the hybrid method (TDMA + NOMA) and TDMA. With TDMA approach the number of sectors was equal to the number of node as described in the Fig. 18. The number of sectors is varying from 2 to 8 , and perform a good fairness than hybrid while the threshold of $4 \mathrm{~dB}$ is applied. The fairness decreases when the number of nodes increases due to the time slot allocation. For a given time $\mathrm{T}$, the time $\mathrm{T}$ is divided into different time slot for each nodes, in that case, the time slot for each node is divided into energy transfer time and information transfer time. In summary, it is proven that our hybrid approach improves the fairness significantly with the increasing number of nodes, as compared to the original TDMA only.

\subsection{Complexity analysis}

The time to performance measurement of the algorithm for calculating sumthroughput can be represented by the Big-O notation. The input values that affect the performance time are the number of nodes $(\mathrm{N})$ and the number of sectors $(\mathrm{M})$. In addition, the sector-specific allocation resource (time) and the transmission rate of each node are constants. Sum-throughput is calculated by the sum of the total transmission rates of each node and the sector-specific allocation resources affected by the input values $\mathrm{N}$ and $\mathrm{M}$. Since $\mathrm{N}$ is quite large especially in case of the IoT environment, $\mathrm{M}$ is negligible compared to $\mathrm{N}$. Thus, according to the Big-O notation, the computational complexity of sum-throughput can be approximated to $\mathrm{O}(\mathrm{N})$. Eventually, it will be appropriate for resource-constrained devices.

\section{Conclusion}

In this paper, we recognized the unfair resource allocation problem of TDMA, which is orthogonal multiple access that can maximize sum-throughput. This unfairness is caused by WPCN's Doubly-Near-Far problem, and it is proposed to mix this difference in power strength with NOMA using the reverse. The proposed hybrid method is based on the assumption that all of the charged energy is used. The optimization problem for maximizing sum-throughput proved to be a convex problem, and as a result, it was possible to efficiently obtain an answer with the same algorithm as the interior-point method. In the proposed method, the existing nodes are grouped in two to form a cluster, so that any number of nodes can be transmitted in the NOMA method. At this time, the performance of the proposed method was analyzed by changing the number of sectors determining the number of nodes using the NOMA method. If the number of sectors is 1, all nodes transmit information at the same time, so it is explained that it is the same MA as TDMA when the 
number of sectors and the number of nodes are the same. The optimal number of sectors must be determined by factors such as application area or quality of service (QoS) to be used because trade off occurs between sum-throughput and fairness. If maximizing sum-throughput is the priority, increase the number of sectors to be close to TDMA and if fairness should be prioritized, reduce the number of sectors to be close to NOMA. Also, it was strongly argued that the difference between SNR should not be used as a condition for SIC, and SINR should be used.

\section{List of Abbreviations}

AP Access Point

CSI Channel State Information

DL Down Link

H-AP Hybrid Access Point

HTT Harvest-Then-Tansmit

IoT Internet of Things

MAC Media Access Control

MA Multiple Access

MISO Multiple Input Single Output

NOMA Non-Orthogonal Multiple Access

PHY Physical layer

QAM Quadrature Amplitude Modulation

QoS Quality of Service

SIC Successive Interference Cancellation

SNIR Signal-to-Noise-plus-Interference Ratio

SNR Signal-to-Noise Ratio

TDMA Time-Division Multiple Access

UL Up Link

WET Wireless Energy Transmission

WIT Wireless Information Transmission

WPCN Wireless Powered Communication Network

WPT Wireless Power Transfer

WSN Wireless Sensor Network

\section{Declarations}

\section{Availability of data and materials}

The datasets used and analysed during the current study are available from the corresponding author on reasonable request.

\section{Competing interests}

The authors declare that they have no competing interests.

\section{Funding}

This work was supported by the National Research Foundation of Korea (NRF) grant funded by the Korea Government (MSIT) (No. NRF-2019R1A2C1009894). 


\section{Authors' contributions}

D.Y., M.K., and J.M. conceived and planned the experiments. D.Y. and M.K. carried out the experiments. D.Y., M.K. and J.M. planned and carried out the simulations. D.Y. wrote the manuscript with support from M.K and J.M. I.J. supervised the project. All authors provided critical feedback and helped shape the research, analysis and manuscript.

\section{Acknowledgements}

Not applicable

\section{Authors Information}

First Author: Mr. Deukgong Yoon, M.S. Student.

Second Author: Mr. Mwamba Kasongo Dahouda, Ph.D. Student.

Third Author: Mr. Juhyun Maeng, Ph.D. Candidate.

Corresponding Author: Dr. Inwhee Joe, Professor.

Author details

Department of Computer Science, Hanynag University, Seoul 04763, South Korea.

References

1. M. Tubaishat and S. Madria, "Sensor networks: an overview," IEEE Potentials, vol. 22, no. 2, pp. 20-23, April-May 2003, doi:10.1109/MP.2003.1197877.

2. S. Bi, Y. Zeng and R. Zhang, "Wireless powered communication networks: an overview," IEEE Wireless Communications, vol. 23, no. 2, pp. 10-18, April 2016, doi: 10.1109/MWC. 2016.7462480.

3. ]D. Niyato, D. In Kim, M. Maso and Z. Han, "Wireless Powered Communication Networks: Research Directions and Technological Approaches," IEEE Wireless Communications, Vol. 24, pp. 88-97, December2017, doi]: 10.1109/MWC.2017.1600116.

4. H. Ju and R. Zhang, "Throughput Maximization in Wireless Powered Communication Networks," IEEE Transactions on Wireless Communications, vol. 13, no. 1, pp. 418-428, January 2014, doi: 10.1109/TWC.2013.112513.130760.

5. Di, X.; Xiong, K.; Fan, P.; Yang, H.-C.; Letaief, K.B. Optimal resource allocation in wirelessly powered communication networks with user cooperation. IEEE Trans. Wirel. Commun. 2017, 16, 7936-7949.

6. Chen, H.; Li, Y.; Rebelatto, J.L.; Ucha-Filho, B.F.; Vucetic, B. Harvest-then-cooperate: Wireless-powered cooperative communications. IEEE Trans. Signal Process. 2015, 63, 1700-1711.

7. Xiong, K.; Chen, C.; Qu, G.; Fan, P.; Letaief, K. Group cooperation with optimal resource allocation in wireless powered communication networks. IEEE Trans. Wirel. Commun. 2017, 16, 3840-3853.

8. Deuk-Gong Yoon, In-Hwi Cho, "Comparison of TDMA vs. NOMA performance according to the distance distribution of user terminals in wireless power communication networks," Fall 2019 Conference presentation, pp.253-254, 2019.11.

9. S. Bi, C. K. Ho, and R. Zhang, "Wireless powered communication: opportunities and challenges," IEEE Communications Magazine, vol. 53, no. 4, pp.117-125, April, 2015.

10. Ju, H.; Zhang, R. User cooperation in wireless powered communication networks. In Proceedings of the 2014 IEEE Global Communications Conference (GLOBECOM), Austin, TX, USA, 8-12 December 2014.

11. FA Rabee, K. Davaslioglu and R. Gitlin, "The optimum received power levels of uplink non-orthogonal multiple access (NOMA) signals," 2017 IEEE 18th Wireless and Microwave Technology Conference (WAMICON), Cocoa Beach, FL, 2017, pp . 1-4, doi: 10.1109/WAMICON.2017.7930242.

12. T. Xie, B. Lyu, Z. Yang, and F. Tian, "User clustering for wireless powered communication networks with non-orthogonal multiple access," IEICE Trans. Fundam. Electron., Commun. Comput. Sci., vol. 101, no. 7, pp. 1146-150, Jul. 2018.

13. L. Liu, R. Zhang, and K. C. Chua, "Multi-antenna wireless powered communication with energy beamforming," IEEE Trans. Commun., vol. 62, no. 12, Dec. 2014, pp. 4349-4361.

14. I.S. Akila, S.V. Manisekaran and R. Venkatesan, "Modern Clustering Techniques in Wireless Sensor Networks," IntechOpen, Oct. 2017, doi:DOI: 10.5772/intechopen.70382.

\section{Figures}


Figure 1 The efficiency of wireless charging method according to distance. There are three kinds of wireless charging methods: inductive coupling (charging by touching within five centimeters $(5 \mathrm{~cm})$, magnetic resonant coupling (charging to a medium distance of 10 to $50 \mathrm{~cm}$ ), and electromagnetic radiation (charging to a distance of more than one meter).

Figure 2 Basic WPCN Model. The basic network of Wireless Powered Communication Network

Figure 3 TDMA frame format in harvest-then-transmit (HTT). Harvest-then-transmit (HTT) protocol was named after the allocation of WET and WIT of time in [3]. After initially transferring energy to the node by WET, it was intended to demonstrate in [3] that each node has a time slot to optimize throughput.

Figure 4 Superposition of signals in signal space. The disparity in power is used to decipher the signal in NOMA. If s1, s2, and s3 signals are obtained at the same time using quadrature amplitude modulation (QAM), they are expressed on the signal constellation.

Figure 5 SIC decoding circuit. The s2 and s3 signals remain after subtracting the approximate s1 from the overall signal. The signal is re-decoded. It's decoded into the first quadrant's sign and it's back in the first quadrant. After that, s2 is measured once more and subtracted from the remaining signal. The residual s3 signal is then decoded, and this signal corresponds to the quadrant; hence, the successive interference cancellation (SIC) process is completed.

Figure 6 Node distribution with small standard deviation. The distance affects and changes the power density, not the orientation; nodes distributed in a spherical form can be placed in a straight line and are distributed next to each other, and the standard deviation of the distance is minimal when expressed quantitatively.

Figure 7 Node distribution with large standard deviation. When the standard deviation is high due to a variety of factors, the NOMA's fairness is high and the TDMA's worth is low.

Figure 8 Capacity region of TDMA and NOMA. Another way to look at this phenomenon is as a capacity region. The intersection of the thick and thin lines determines the $R 1$ and $R 2$ values. It can be assumed that $C D$ is based on it. The values of $R 1$ and $R 2$ are not equal in this case. However, if NOMA is correctly implemented, there is a potential probability of getting throughput anywhere between $C$ and $B$, which increases fairness.

Figure 9 System model. The nodes are randomly distributed in a circular shape around the H-AP.

Figure 10 Harvest-then-transmit protocol with digital beamforming. T1 and T2 are the lengths of WET and WIT in one frame, and each $k$ is the DL and UL time per sector. The duration of the WET is determined by H-AP using the time allocated to the sector with the maximum transmission rate.

Figure 11 Nodes distributed Randomly. The efficiency of Hybrid TDMA and NOMA proposed in this paper was calculated when the number of nodes was 100 and the number of sectors was increased from 1 to 15 . One circle points to one node, while the middle triangle points to the H-AP. The maximum sum-throughput is computed per time, the node structure is uniformly redistributed, and the operation is replicated 500 times to average the results. 
Figure 12 Comparison of Sum-Throughput according to the number of sectors without applying SINR threshold. When we first look at the maximum sum-throughput, we can see that it remains constant regardless of the number of sectors. This value is consistent with HTT's result, indicating that the maximum sum-throughput is technically the same.

Figure 13 Three fairness according to the number of sectors. The fairness in between-sector, the fairness in-sector, and the fairness of all-node was measured.

Figure 14 Sum-Throughput according to WET power and number of sectors $=5$. The simulation results vary as the threshold is applied; the number of nodes is reduced to 30 , and if the number of nodes is too high, the likelihood of decoding increases.

Figure 15 Sum-Throughput according to WET power and number of sectors $=15$. When there are 5 sector, even a SINR threshold of around $3 \mathrm{~dB}$ results in a noticeable reduction in throughput relative to when there is no threshold; the higher the threshold value, the greater the reduction.

Figure 16 Performance comparison by applying threshold and changing number of sectors: fairness measured between sectors. The sum-throughput and fairness trade-off exists since, as the number of sectors grows, the sum-throughput grows but the fairness decreases.

Figure 17 Comparison of fairness between clustering method and sector method: fairness measured for all nodes. the fairness was calculated by the method proposed in the paper, and the value was slightly smaller.

Figure 18 Comparison of fairness between Hybrid TDMA + NOMA and TDMA based on number of sectors and nodes: fairness measured between sectors. The number of sectors is varying from 2 to 8 , and perform a good fairness than hybrid while the threshold of $4 \mathrm{~dB}$ is applied 
Figures

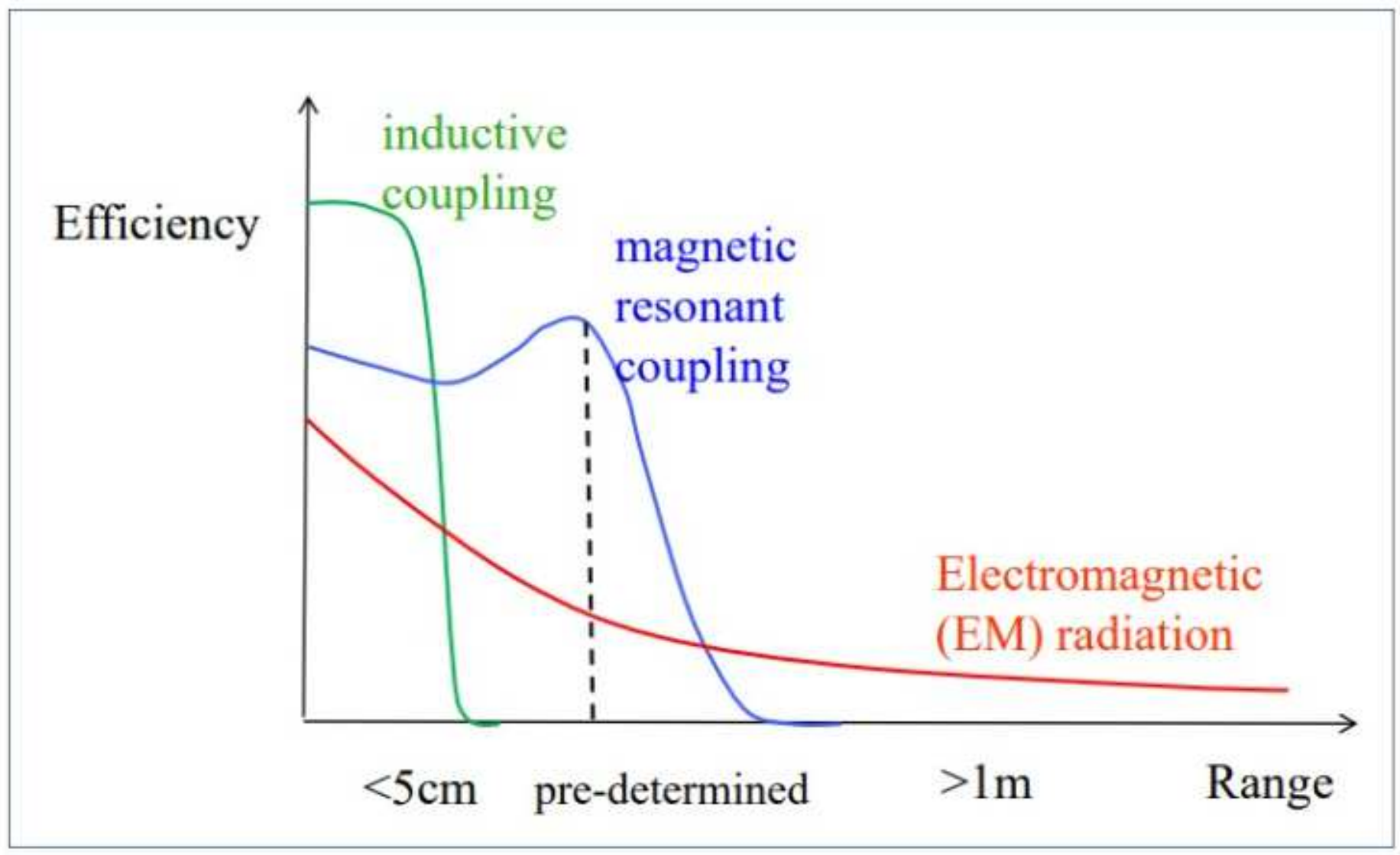

\section{Figure 1}

The efficiency of wireless charging method according to distance. There are three kinds of wireless charging methods: inductive coupling (charging by touching within five centimeters $(5 \mathrm{~cm})$, magnetic resonant coupling (charging to a medium distance of 10 to $50 \mathrm{~cm}$ ), and electromagnetic radiation (charging to a distance of more than one meter). 


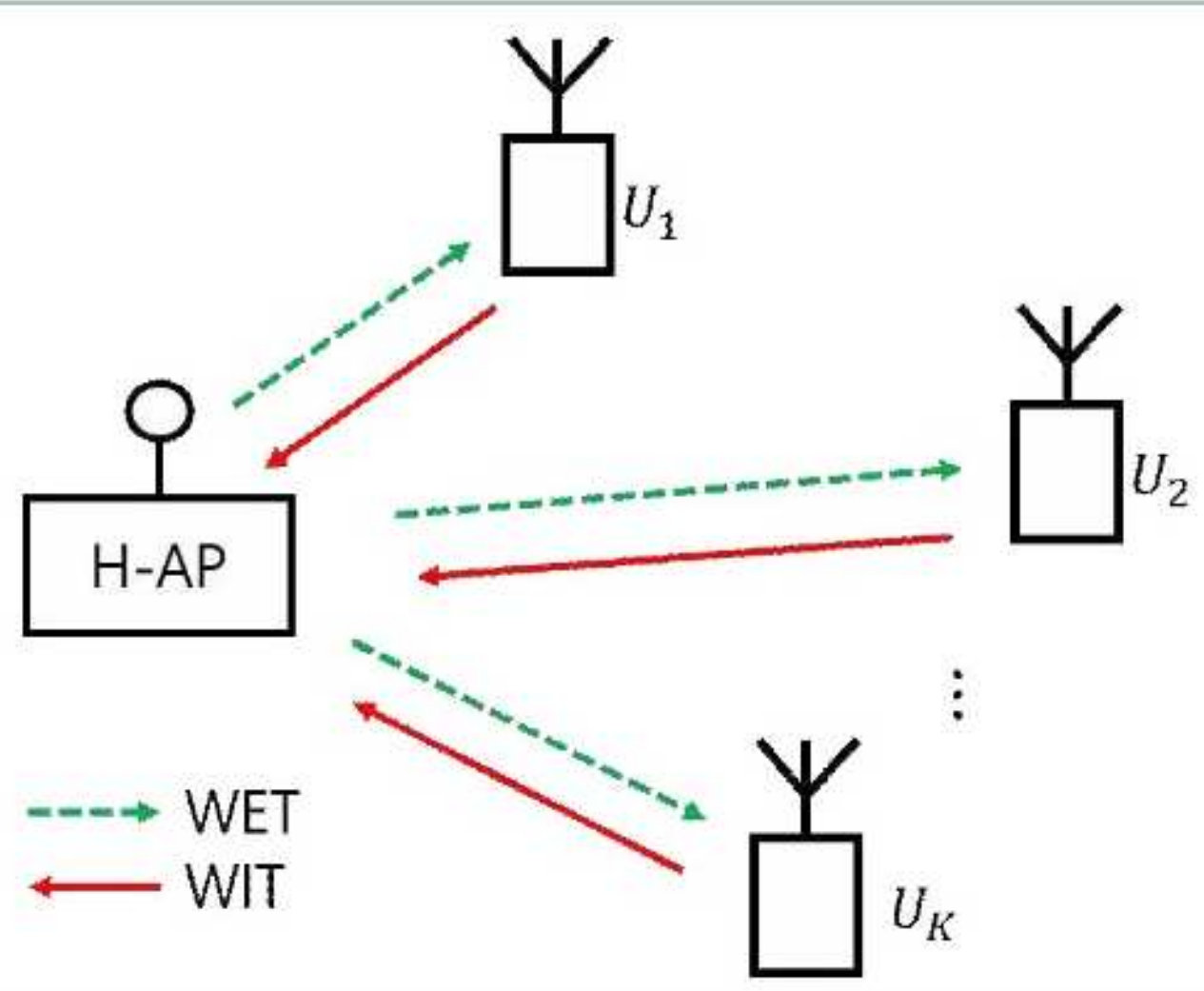

Figure 2

Basic WPCN Model. The basic network of Wireless Powered Communication Network

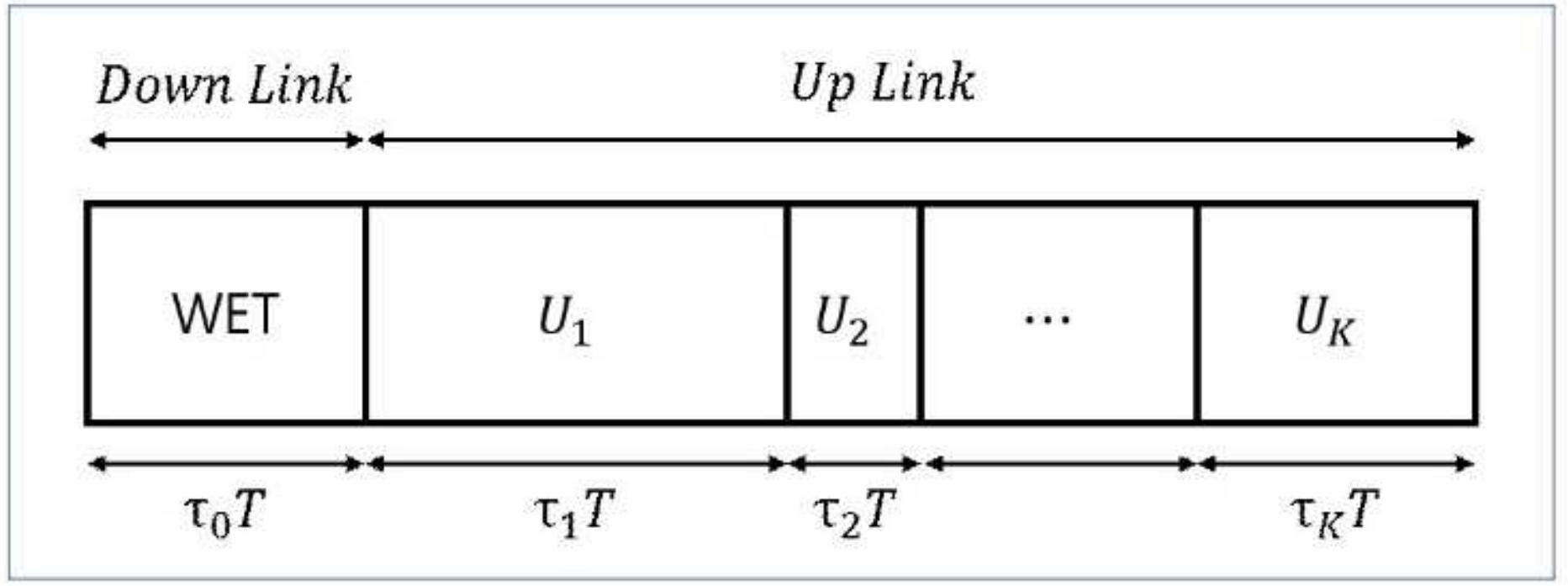

Figure 3

TDMA frame format in harvest-then-transmit (HTT). Harvest-then-transmit (HTT) protocol was named after the allocation of WET and WIT of time in [3]. After initially transferring energy to the node by WET, it was intended to demonstrate in [3] that each node has a time slot to optimize throughput. 


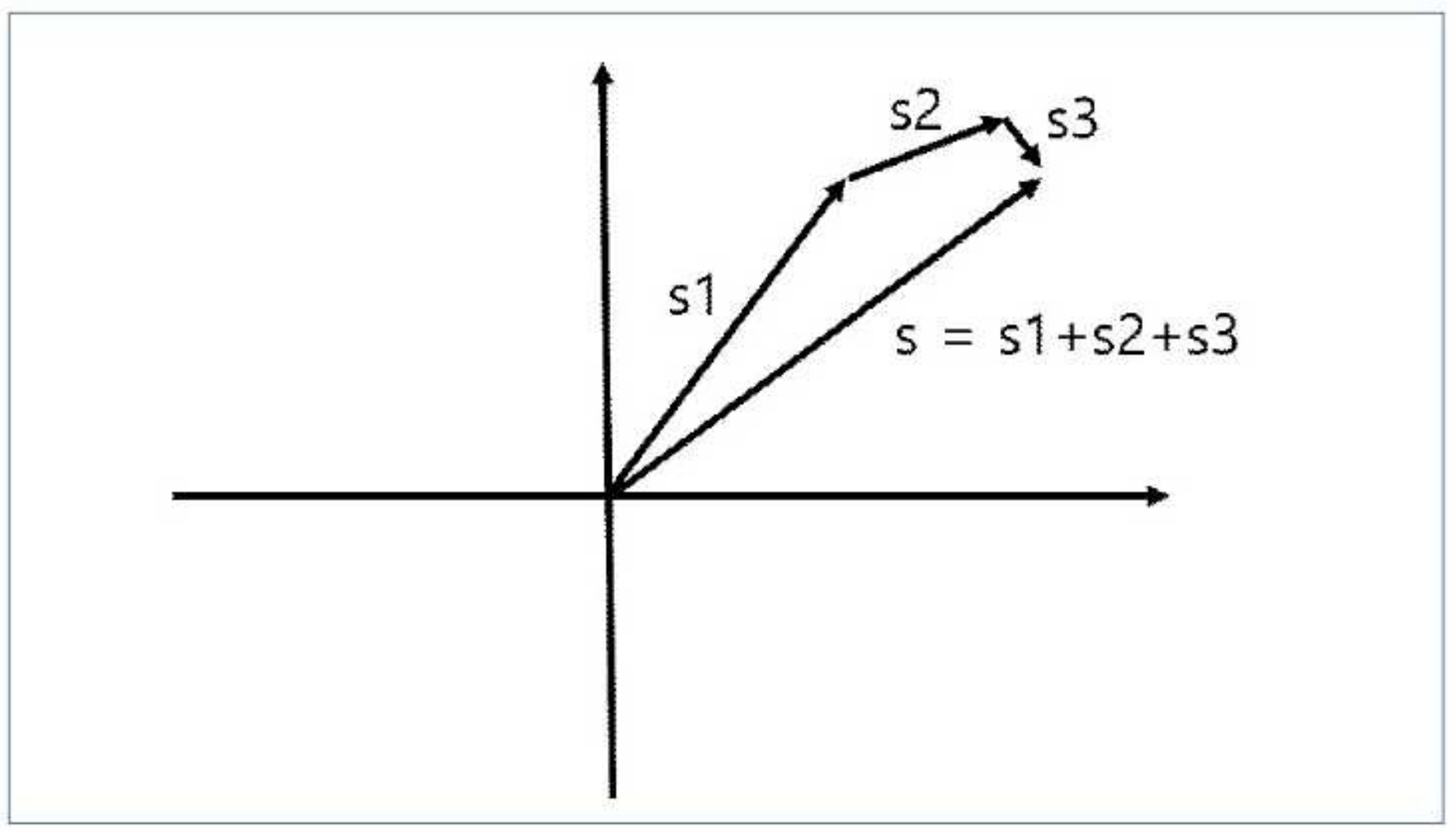

Figure 4

Superposition of signals in signal space. The disparity in power is used to decipher the signal in NOMA. If s1, s2, and s3 signals are obtained at the same time using quadrature amplitude modulation (QAM), they are expressed on the signal constellation.

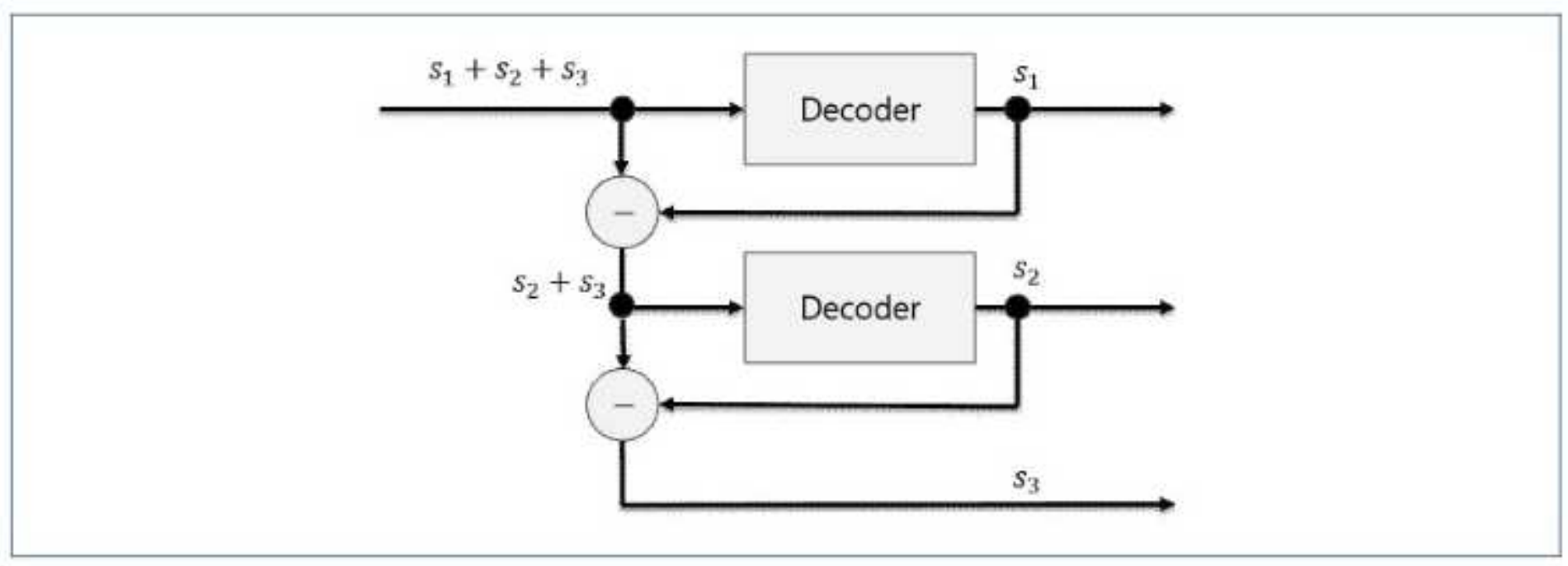

Figure 5

SIC decoding circuit. The $s 2$ and $s 3$ signals remain after subtracting the approximate $s 1$ from the overall signal. The signal is re-decoded. It's decoded into the first quadrant's sign and it's back in the first 
quadrant. After that, $s 2$ is measured once more and subtracted from the remaining signal. The residual s 3 signal is then decoded, and this signal corresponds to the quadrant; hence, the successive interference cancellation (SIC) process is completed.
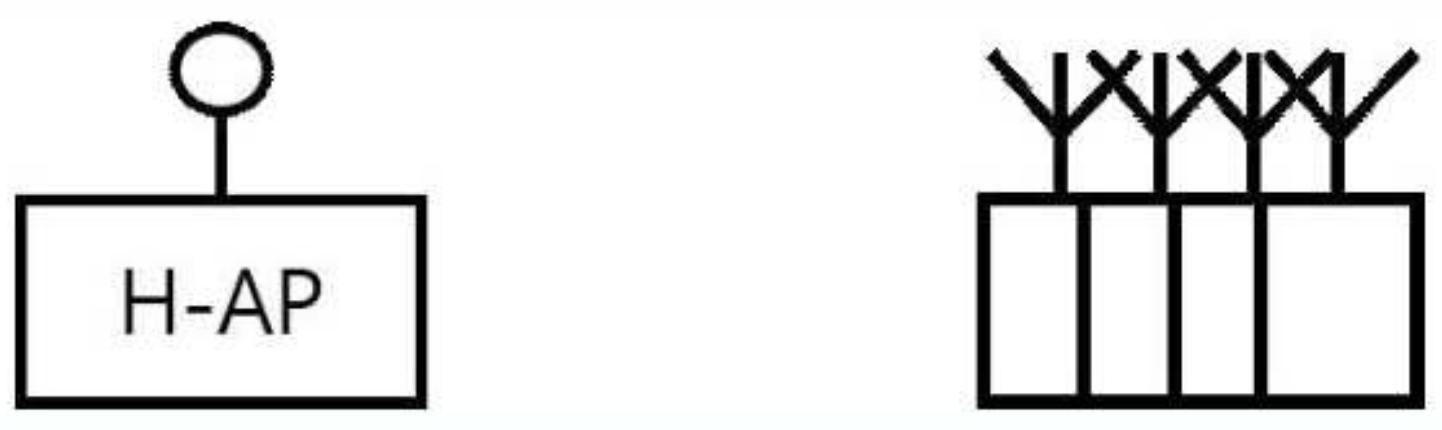

\section{Figure 6}

Node distribution with small standard deviation. The distance affects and changes the power density, not the orientation; nodes distributed in a spherical form can be placed in a straight line and are distributed next to each other, and the standard deviation of the distance is minimal when expressed quantitatively.
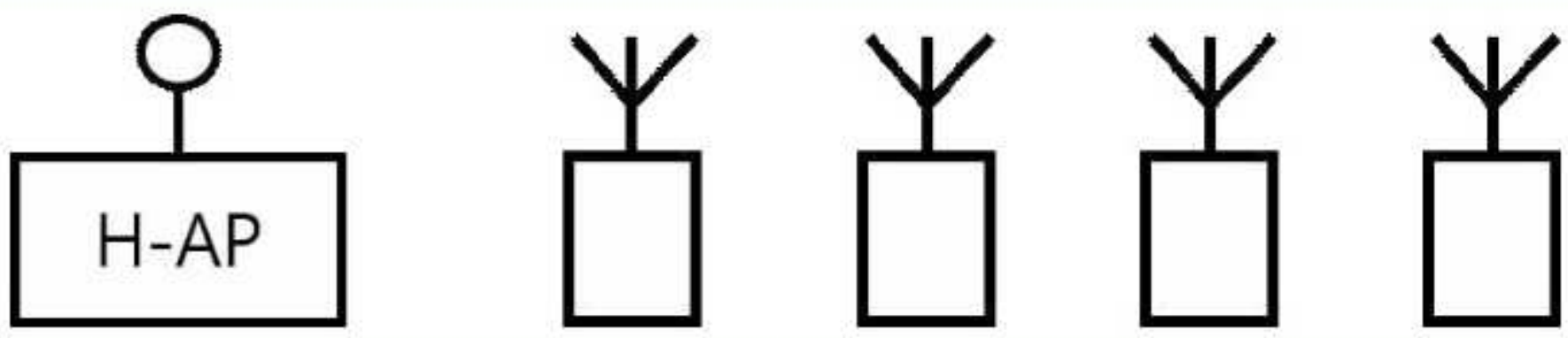

\section{Figure 7}

Node distribution with large standard deviation. When the standard deviation is high due to a variety of factors, the NOMA's fairness is high and the TDMA's worth is low. 


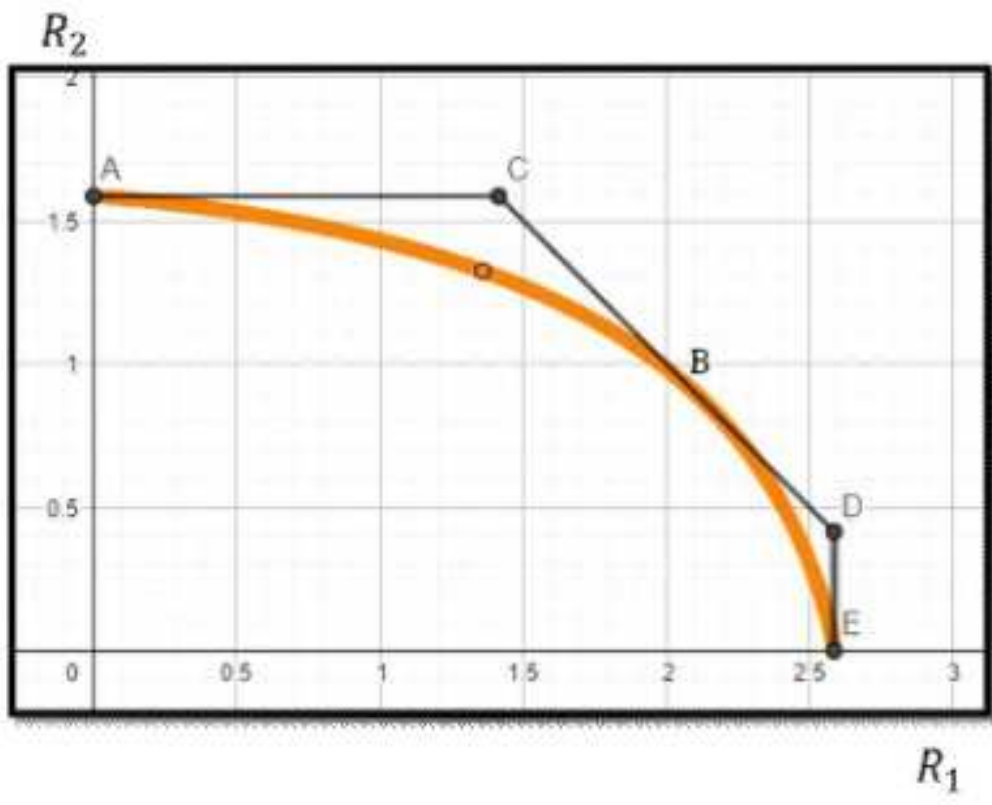

\section{Figure 8}

Capacity region of TDMA and NOMA. Another way to look at this phenomenon is as a capacity region. The intersection of the thick and thin lines determines the R1 and R2 values. It can be assumed that CD is based on it. The values of R1 and R2 are not equal in this case. However, if NOMA is correctly implemented, there is a potential probability of getting throughput anywhere between $C$ and $B$, which increases fairness. 


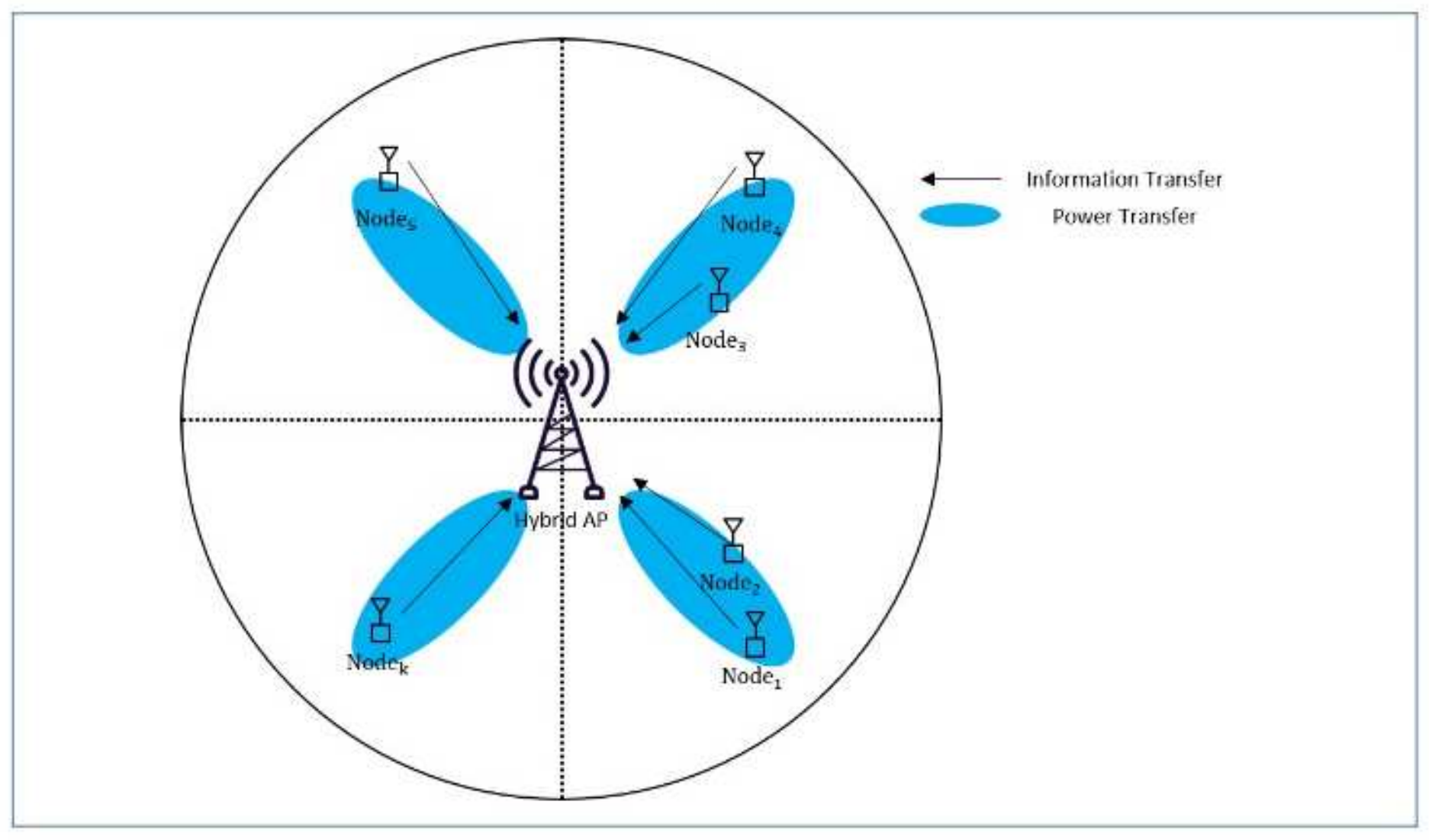

Figure 9

System model. The nodes are randomly distributed in a circular shape around the H-AP.

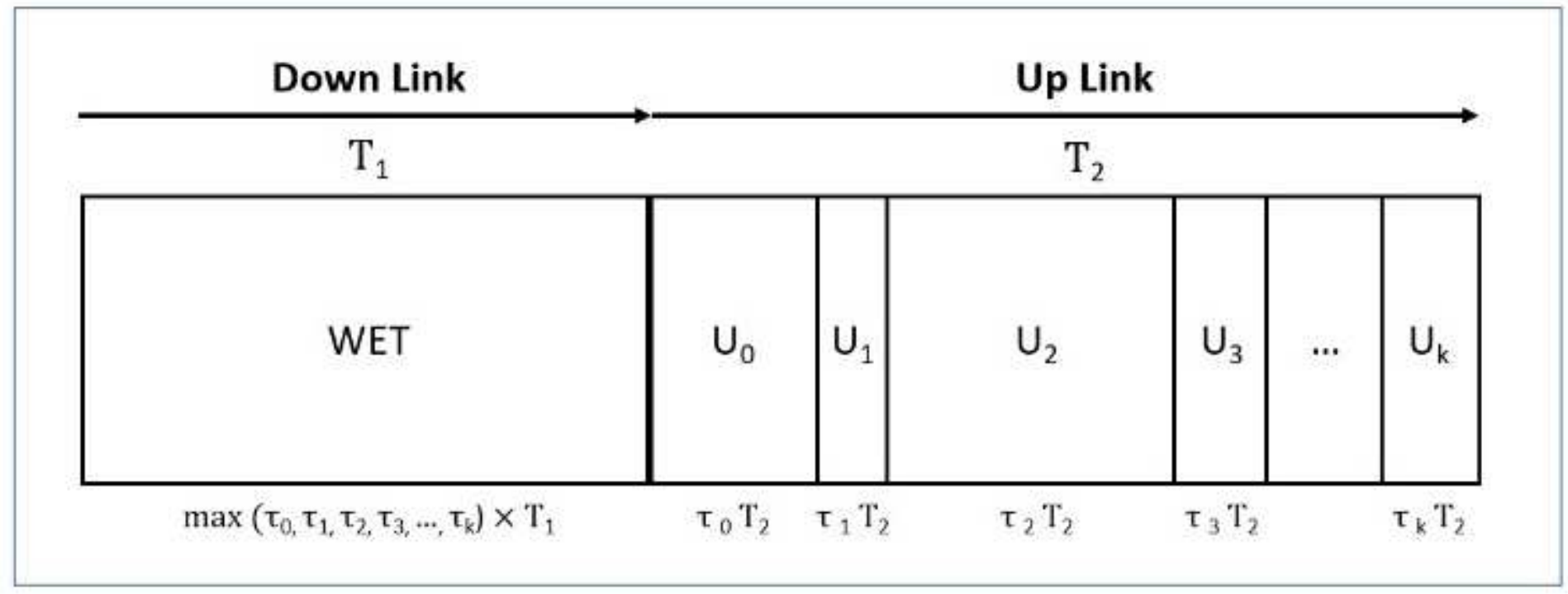

Figure 10

Harvest-then-transmit protocol with digital beamforming. T1 and T2 are the lengths of WET and WIT in one frame, and each $\mathrm{k}$ is the DL and UL time per sector. The duration of the WET is determined by H-AP using the time allocated to the sector with the maximum transmission rate. 


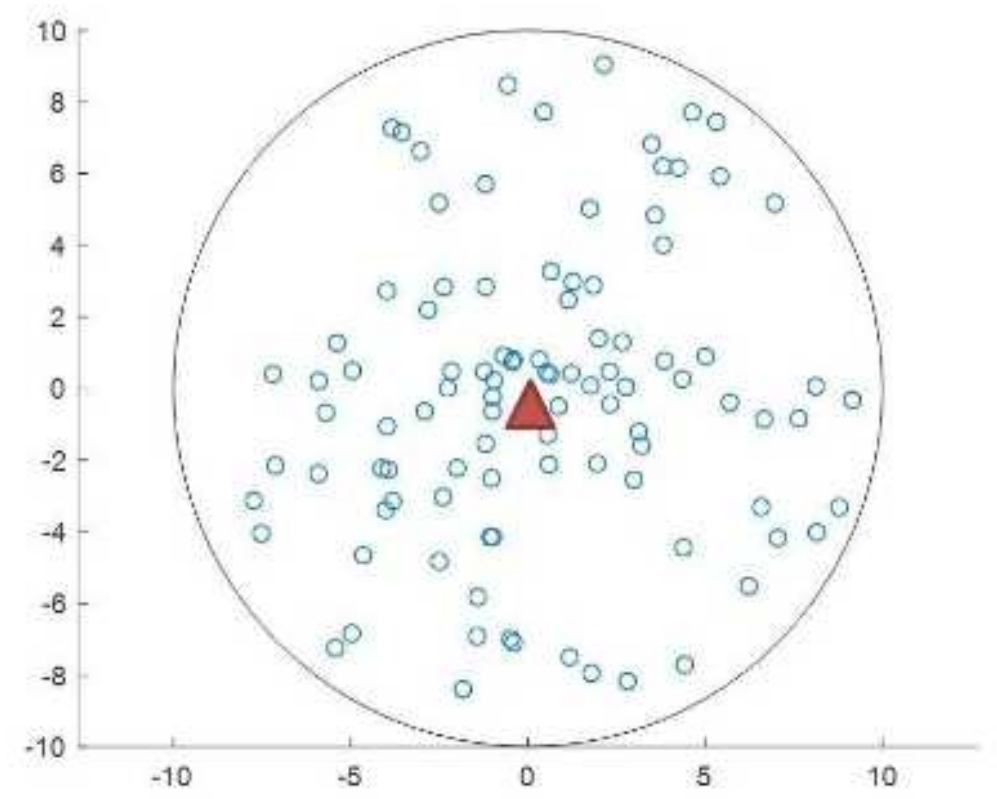

\section{Figure 11}

Nodes distributed Randomly. The effciency of Hybrid TDMA and NOMA proposed in this paper was calculated when the number of nodes was 100 and the number of sectors was increased from 1 to 15 . One circle points to one node, while the middle triangle points to the H-AP. The maximum sum-throughput is computed per time, the node structure is uniformly redistributed, and the operation is replicated 500 times to average the results. 


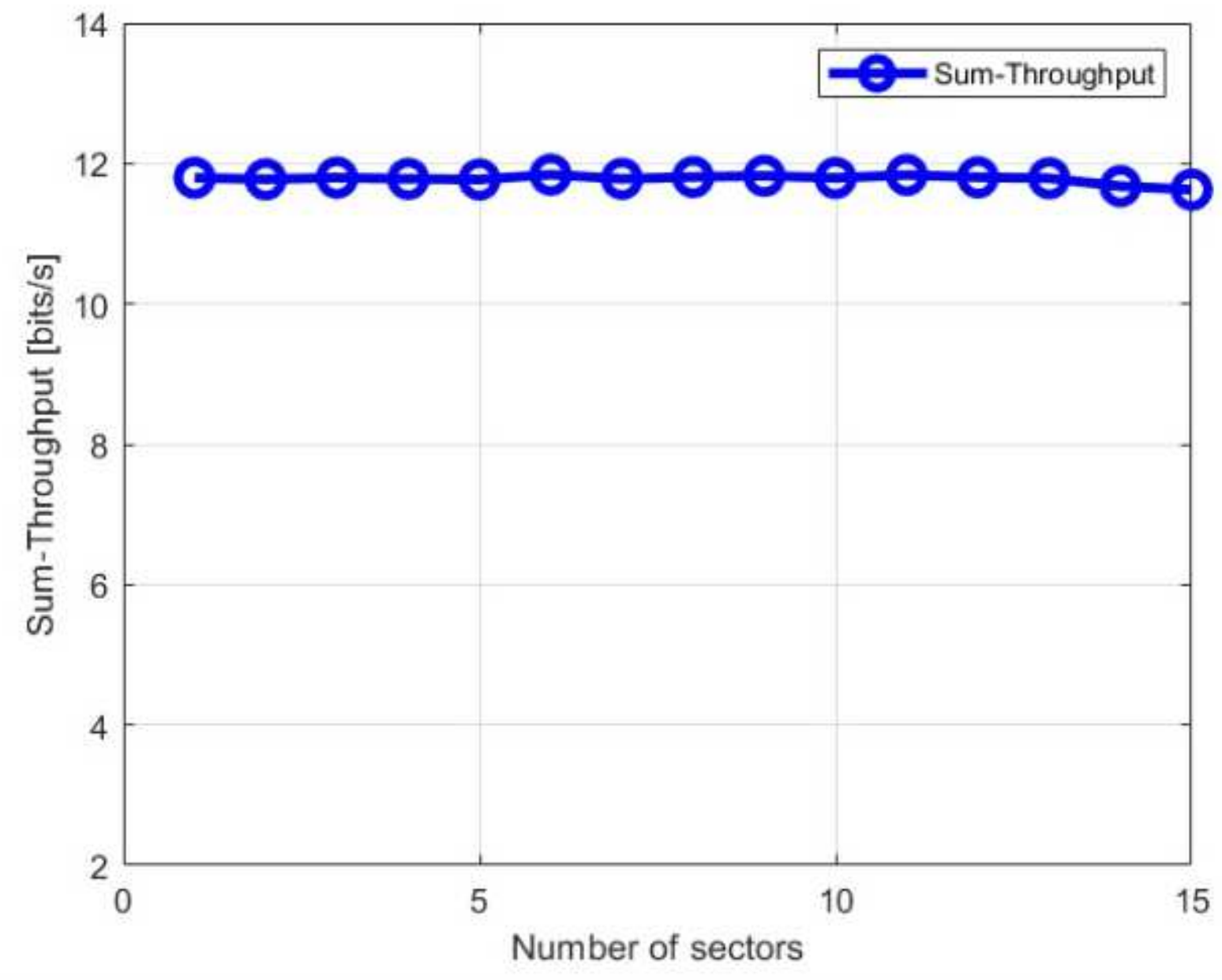

Figure 12

Comparison of Sum-Throughput according to the number of sectors without applying SINR threshold. When we first look at the maximum sum-throughput, we can see that it remains constant regardless of the number of sectors. This value is consistent with HTT's result, indicating that the maximum sumthroughput is technically the same. 


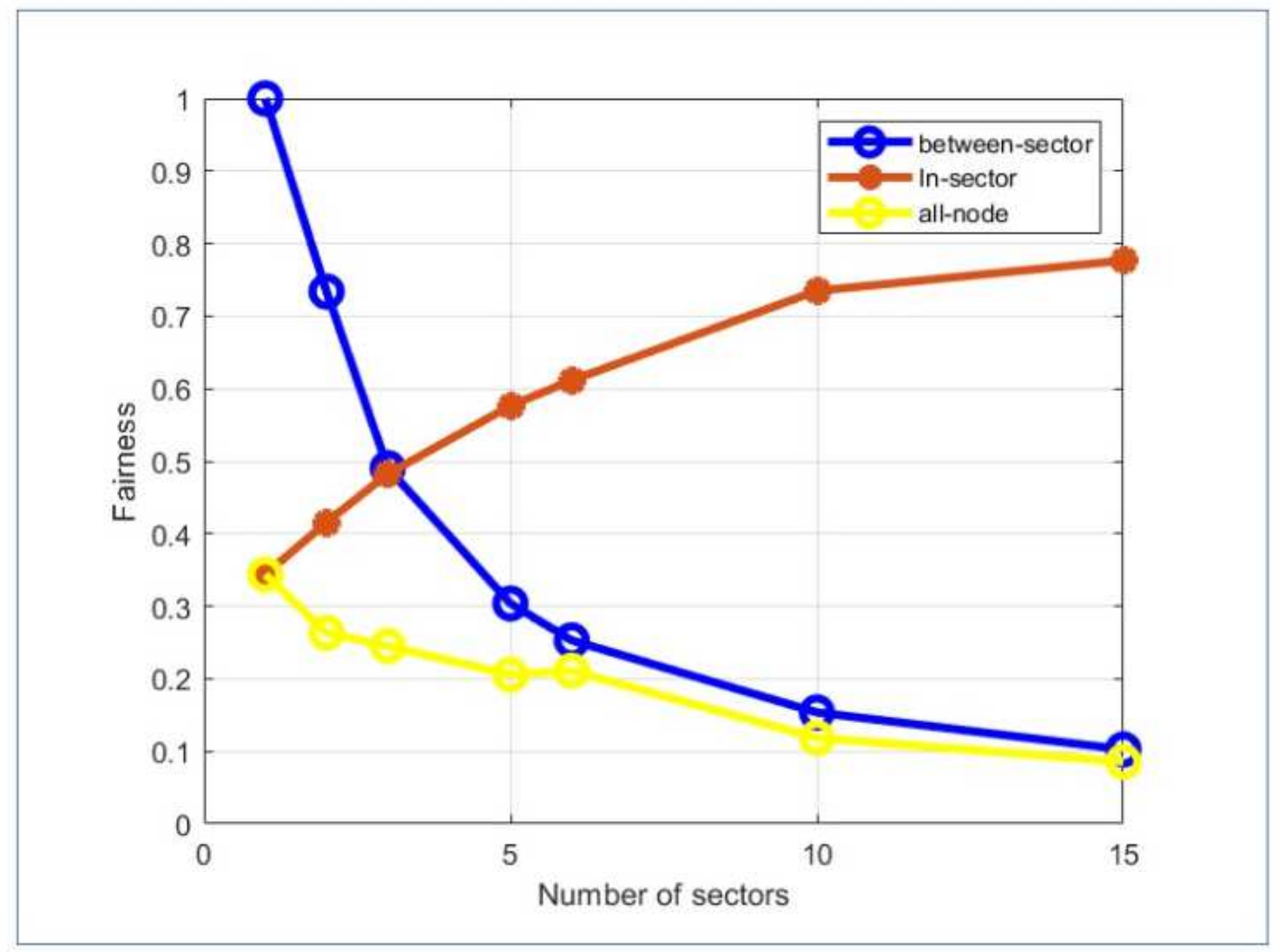

Figure 13

Three fairness according to the number of sectors. The fairness in between-sector, the fairness in-sector, and the fairness of all-node was measured. 


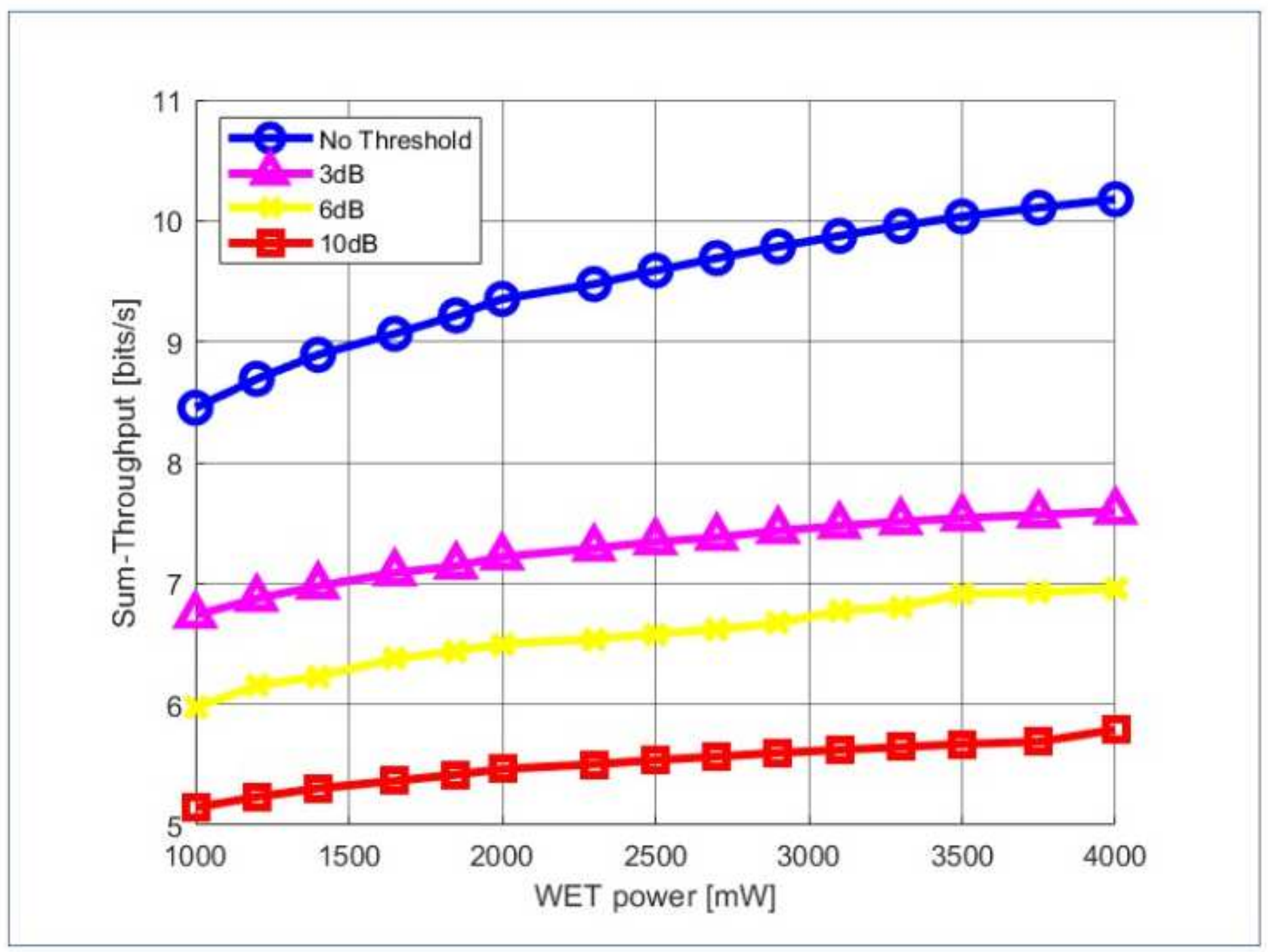

Figure 14

Sum-Throughput according to WET power and number of sectors $=5$. The simulation results vary as the threshold is applied; the number of nodes is reduced to 30 , and if the number of nodes is too high, the likelihood of decoding increases. 


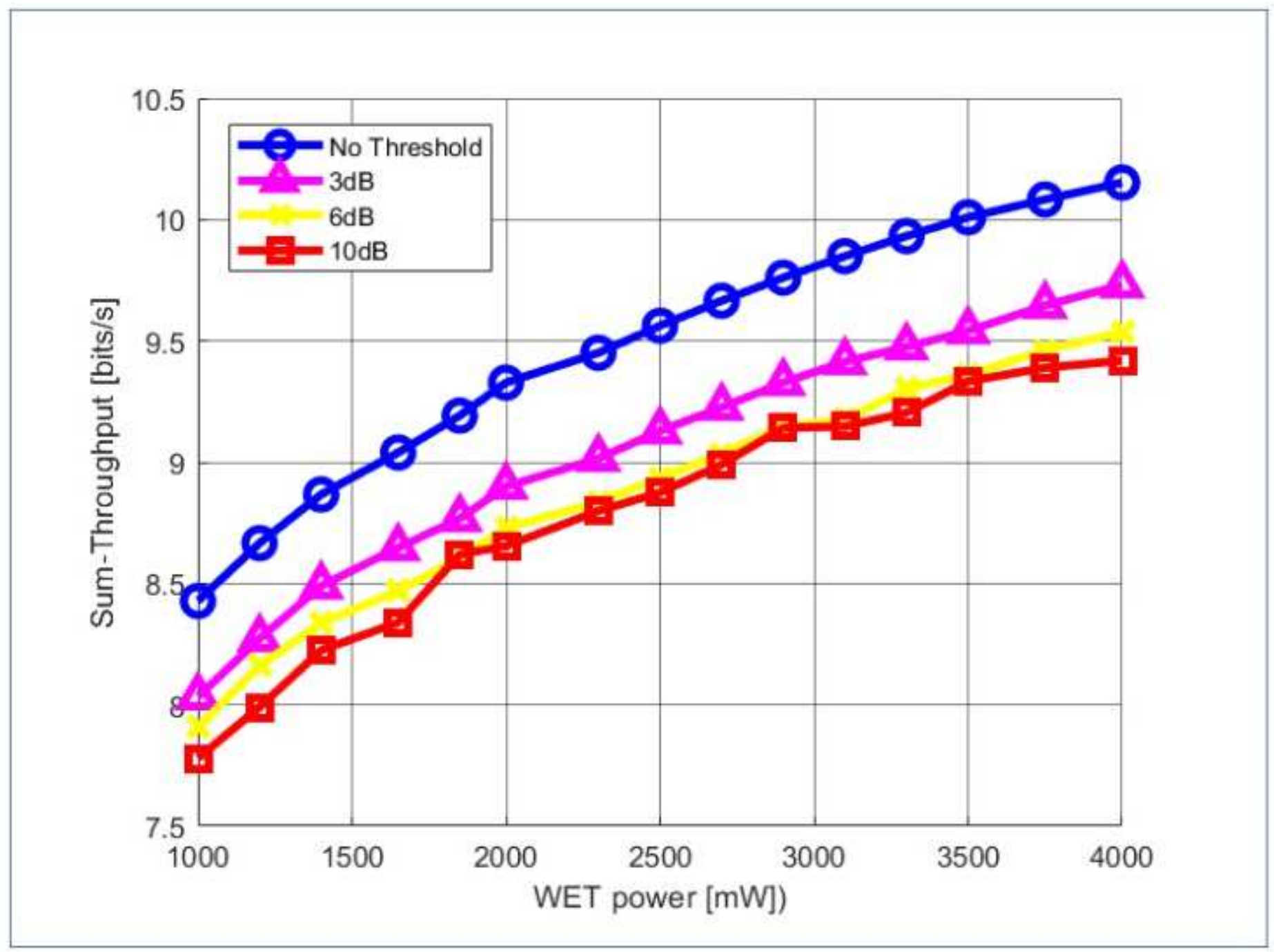

Figure 15

Sum-Throughput according to WET power and number of sectors $=15$. When there are 5 sector, even a SINR threshold of around $3 \mathrm{~dB}$ results in a noticeable reduction in throughput relative to when there is no threshold; the higher the threshold value, the greater the reduction. 


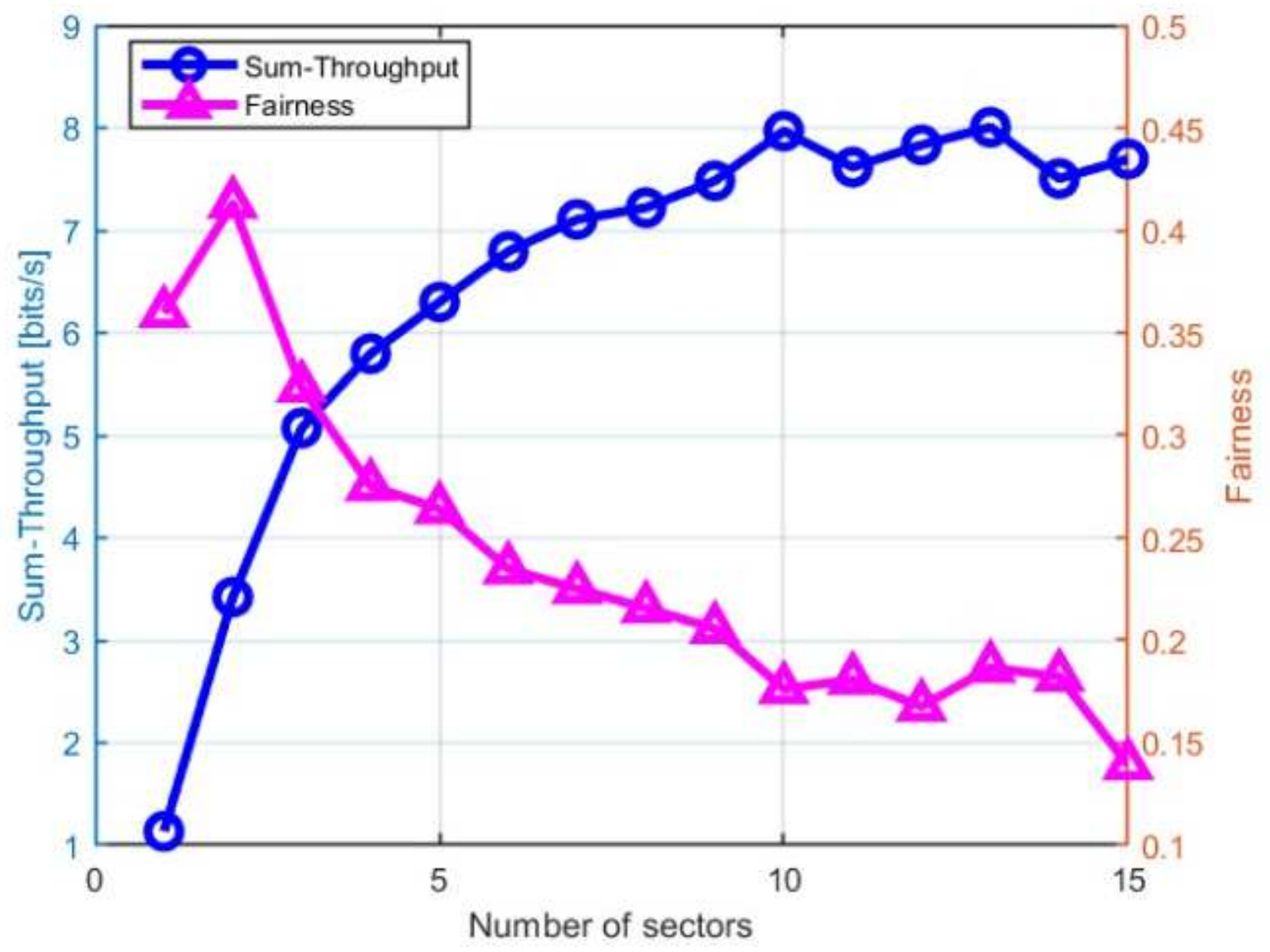

Figure 16

Performance comparison by applying threshold and changing number of sectors: fairness measured between sectors. The sum-throughput and fairness trade-off exists since, as the number of sectors grows, the sum-throughput grows but the fairness decreases. 


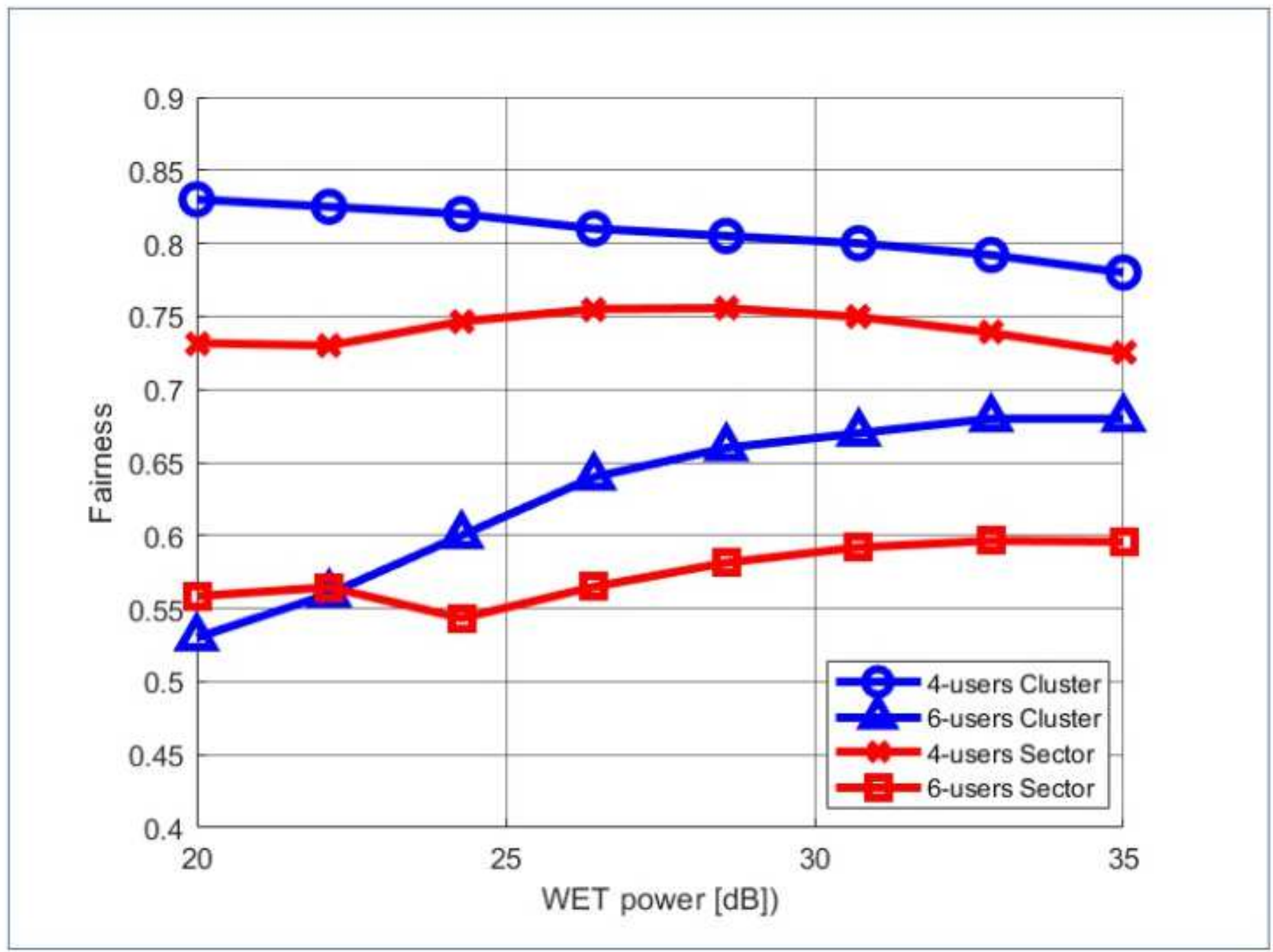

Figure 17

Comparison of fairness between clustering method and sector method: fairness measured for all nodes. the fairness was calculated by the method proposed in the paper, and the value was slightly smaller. 


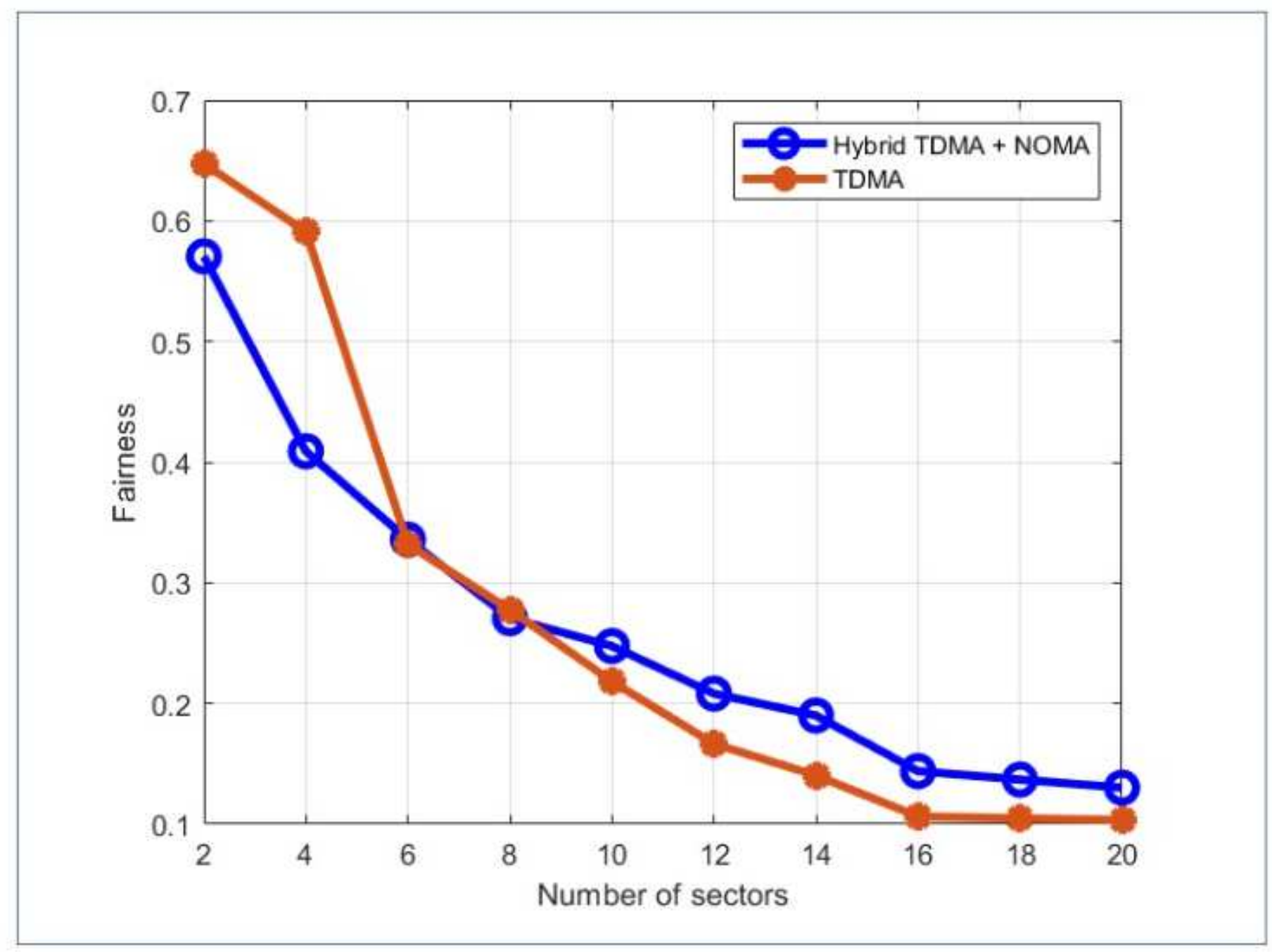

Figure 18

Comparison of fairness between Hybrid TDMA + NOMA and TDMA based on number of sectors and nodes: fairness measured between sectors. The number of sectors is varying from 2 to 8 , and perform a good fairness than hybrid while the threshold of $4 \mathrm{~dB}$ is applied 